\title{
Advection-dominated Accretion: Underfed Black Holes and Neutron Stars
}

\section{Citation}

Narayan, Ramesh, and Insu Yi. 1995. "Advection-Dominated Accretion: Underfed Black Holes and Neutron Stars." The Astrophysical Journal 452 (October): 710. https:// doi.org/10.1086/176343.

\section{Permanent link}

http://nrs.harvard.edu/urn-3:HUL.InstRepos:41384896

\section{Terms of Use}

This article was downloaded from Harvard University's DASH repository, and is made available under the terms and conditions applicable to Other Posted Material, as set forth at http:// nrs.harvard.edu/urn-3:HUL.InstRepos:dash.current.terms-of-use\#LAA

\section{Share Your Story}

The Harvard community has made this article openly available.

Please share how this access benefits you. Submit a story.

Accessibility 


\title{
Advection-Dominated Accretion: Underfed Black Holes and Neutron Stars
}

\author{
Ramesh Narayan and Insu Yi \\ Harvard-Smithsonian Center for Astrophysics \\ 60 Garden Street, Cambridge, MA 02138
}

\begin{abstract}
We describe new optically thin solutions for rotating accretion flows around black holes and neutron stars. These solutions are advection-dominated, so that most of the viscously dissipated energy is advected radially with the flow. We model the accreting gas as a twotemperature plasma and include cooling by bremsstrahlung, synchrotron, and Comptonization. We obtain electron temperatures $T_{e} \sim 10^{8.5}-10^{10} \mathrm{~K}$.

The new solutions are present only for mass accretion rates $\dot{M}$ less than a critical rate $\dot{M}_{c r i t}$ which we calculate as a function of radius $R$ and viscosity parameter $\alpha$. For $\dot{M}<\dot{M}_{\text {crit }}$ we show that there are three equilibrium branches of solutions. One of the branches corresponds to a cool optically thick flow which is the well-known thin disk solution of Shakura \& Sunyaev (1973). Another branch corresponds to a hot optically thin flow, discovered originally by Shapiro, Lightman \& Eardley (1976, SLE). This solution is thermally unstable. The third branch corresponds to our new advection-dominated solution. This solution is hotter and more optically thin than the SLE solution, but is viscously and thermally stable. It is related to the ion torus model of Rees et al. (1982) and may potentially explain the hard X-ray and $\gamma$-ray emission from X-ray binaries and active galactic nuclei.

For $\dot{M}<\dot{M}_{\text {crit }}$, our work suggests that an accretion flow can choose between two distinct states, namely the thin disk solution and the new advection-dominated solution, both of which are apparently stable. We argue that, in certain circumstances, it is only the latter solution that is truly stable, and that a thin disk will spontaneously evaporate and convert itself into an advection-dominated flow. Even for $\dot{M}>\dot{M}_{\text {crit }}$ we suggest that a thin disk may evaporate partially so that a fraction of the accretion occurs via an advectiondominated hot corona. If these ideas are correct, then optically thin advection-dominated flows must be very widespread, possibly the most common form of accretion in black holes accreting at sub-Eddington $\dot{M}$.

Our calculations indicate that advection-dominated accretion on black holes differs considerably from similar flows around neutron stars. The crucial physical difference, which has been previously mentioned in the literature, is that in the former the advected energy is lost into the hole whereas in the latter it is thermalized and reradiated at the stellar surface, thereby providing soft photons which can Compton-cool the accreting gas. We obtain $\dot{M}_{c r i t} \sim \alpha^{2} \dot{M}_{E d d}$ for accreting black holes, independent of the black hole mass, whereas it is $\sim 0.1 \alpha^{2} \dot{M}_{E d d}$ for neutron stars. Advection-dominated accretion is therefore more likely to occur in accreting black holes, and these systems will be underluminous for their $\dot{M}$ because the bulk of the energy is advected into the hole rather than being radiated. We find that $T_{e}$ in accreting black hole flows rises up to $\sim 10^{9}-10^{10} \mathrm{~K}$, compared to $T_{e} \sim 10^{8.5}-10^{9} \mathrm{~K}$ in neutron star systems. Spectra of accreting black holes are therefore expected to be harder
\end{abstract}


than those of accreting neutron stars. Pair effects are also more likely in black hole systems, though only at higher $\dot{M}$ than those we consider.

Subject headings: accretion, accretion disks, black hole physics, galaxies: nuclei, quasars, hydrodynamics, stars: neutron, X-rays: stars 


\section{Introduction}

One of the cornerstones of the theory of accretion disks is the model of thin disks developed by Shakura \& Sunyaev (1973), Novikov \& Thorne (1973), and Lynden-Bell \& Pringle (1974) (see Frank, King \& Raine 1992 for a review). This model, which has been widely applied to many accreting systems, is based on the assumption that the accreting material cools efficiently so that all the energy which is released through viscosity is radiated locally. As a consequence, the accreting gas is much cooler than the local virial temperature, and therefore the orbiting material has a vertical thickness which is much smaller than the radius.

What happens if the cooling in an accretion flow is not efficient? Clearly, if the cooling is unable to keep up with the heating, then a part of the viscously released energy will have to be advected with the accreting gas as stored entropy. We may then expect the gas to be driven to a higher temperature, which will lead to a vertical thickening of the disk. Pressure forces also will become important and will modify the dynamics; for instance, the rotation is likely to be sub-Keplerian. Accretion flows with some of these properties have been considered from time to time in various contexts (e.g. Begelman 1978, Liang \& Thompson 1980, Paczyński \& Wiita 1980, Phinney 1981, Rees et al. 1982, Begelman \& Meier 1982, Liang 1988, Eggum, Coroniti \& Katz 1988, Abramowicz et al. 1988, Narayan \& Yi 1994, 1995, hereafter Papers 1, 2).

The basic dynamical equations of accretion with inclusion of entropy advection have been written by various workers, notably Paczyński \& Bisnovatyi-Kogan (1981) and Muchotrzeb \& Paczyński (1982) who derived a particular set of equations using a simplifying height-integration approximation. The formalism was developed further by Abramowicz et al. (1988), who named the approach the "slim disk" model, and carried out a comprehensive analysis of accretion flows around black holes with mass accretion rates $\dot{M}$ comparable to or greater than the Eddington rate. These authors discovered a new solution branch at super-Eddington $\dot{M}$ where the optical depth of the accreting gas is so high that the diffusion time becomes longer than the viscous time (see Begelman 1978 for an early discussion of this kind of radiation trapping). Consequently, the gas is unable to cool and instead advects the dissipated energy. The new advection-dominated solution of Abramowicz et al. (1988) is both thermally and viscously stable.

Following on the work of Abramowicz et al. (1988), the slim disk model has been applied to the study of thermal and viscous instabilities and limit cycles in optically thick accretion disks (e.g. Honma, Matsumoto \& Kato 1991, Wallinder 1991, Chen \& Taam 1993). It has also been used successfully in the modeling of boundary layers (Narayan \& Popham 1993, Popham et al. 1993, Popham \& Narayan 1995).

Beginning with the pioneering work of Shapiro, Lightman \& Eardley (1976, hereafter SLE), investigations of black hole accretion disks at lower $\dot{M}$ have focused on a class of optically thin solutions where the gas is significantly hotter than the local Shakura-Sunyaev solution. The accreting plasma in these solutions is two-temperature, with the ions being significantly hotter than the electrons. Since the gas is optically thin, the cooling occurs through bremsstrahlung or via Comptonization of the bremsstrahlung photons and other soft photons that may be produced locally. These hot disk models have been applied to the various states of Cyg X-1 (SLE 1976, Melia \& Misra 1994), while a related model, the "ion torus" (Rees et al. 1982), has been applied to active galactic nuclei (AGN) at low $\dot{M}$. Recently, Wandel \& Liang (1991) and Luo \& Liang (1994) introduced a phenomenological 
bridging formula in the radiative transfer equations to display the topological relationship between the SLE hot solution and the Shakura-Sunyaev thin disk. Kusunose \& Takahara (1989) considered the effects of electron-positron pairs, magnetic fields and cyclotron emission.

A major attraction of the SLE branch of solutions is that the plasma attains electron temperatures $T_{e} \sim 10^{9} \mathrm{~K}$, so that these solutions have the promise of being able to explain the hard X-ray and $\gamma$-ray emission seen in X-ray binaries and AGN. Unfortunately, the solutions are thermally unstable (though they are viscously stable). The thermal instability arises because the accreting gas is optically thin so that the cooling efficiency via bremsstrahlung decreases with decreasing density (Pringle, Rees \& Pacholczyk 1973, Piran 1978). Because of this feature, if an equilibrium SLE flow is perturbed to a slightly higher temperature, its density goes down and the rate of cooling decreases. This causes the gas to heat up further, leading to a runaway thermal instability. Kusunose \& Takahara (1989) showed that the inclusion of pairs in the physics of the hot plasma does not remove the instability.

What is the fate of an optically thin flow which encounters the thermal instability? In analogy with the Abramowicz et al. (1988) work on optically thick flows described above, one might suspect that optically thin flows may again have a hitherto undiscovered solution in which entropy advection dominates. If such an advection-dominated solution were present, it would be even hotter and more optically thin than the SLE solution, and it is likely to be stable. Surprisingly, this point does not seem to have been fully appreciated until very recently. There is a suggestion of these ideas in the ion torus paper of Rees et al. (1982). However, the first real hint that advection can introduce stability in optically thin flows came with the work of Narayan \& Popham (1993), who studied boundary layers in cataclysmic variables (CVs) and found that at low $\dot{M}$ the boundary layer becomes optically thin and thermally unstable. However, the instability does not affect the overall flow significantly, since the accreting material just switches to a local advection-dominated state in the optically thin zone, a point emphasized more recently by Abramowicz et al. (1995).

Following up on the Narayan \& Popham (1993) work, the present authors investigated in Papers 1 and 2 the general properties of advection-dominated flows. In these papers we derived self-similar solutions, both of the slim disk equations (Paper 1) and of a more general non-height-integrated set of equations (Paper 2). In earlier work, Spruit et al. (1987) considered self-similar height-integrated solutions in the context of the spiral-shock wave driven instability and found that for low values of the ratio of specific heats accretion without radiative losses is possible. The solutions in Papers 1 and 2 give us quantitative estimates of the angular velocity, radial velocity, pressure, temperature, etc. of an advectiondominated accretion flow as functions of a few basic parameters, such as the ratio of specific heats $\gamma$ of the gas and the viscosity parameter $\alpha$. More importantly, the solutions reveal that advection-dominated flows have qualitatively very different properties than standard thin accretion disks: (1) advection-dominated flows are almost spherical in their morphology (not at all disk-like); (2) they do not have empty funnels (as some workers have suggested they might); (3) they rotate quite slowly compared to Keplerian (tending to zero rotation as $\gamma \rightarrow 5 / 3$ ); (4) the radial flow velocities are comparable to the free fall velocity (therefore accretion occurs much more rapidly than in a thin disk); (5) the flows are convectively unstable (which would contribute at least partially to the viscosity); (6) they often have a positive Bernoulli constant (which may lead to the formation of jets and outflows); and (7) by definition, advection-dominated flows radiate much less efficiently than thin disks, and therefore are ultra-dim for their accretion rate (Phinney 1981, Rees et al. 1982). 
In Papers 1 and 2 we discussed the two relevant limits under which an accretion flow becomes advection-dominated, namely at very high accretion rates, where the optical depth of the gas is very large (Begelman 1978, Abramowicz et al. 1988), and at low accretion rates, where the optical depth of the gas becomes significantly less than unity (Rees et al. 1982, Narayan \& Popham 1993, Abramowicz et al. 1995). However, lacking a detailed model, we could not determine whether or not advection-dominated accretion is truly relevant to real systems in nature. We investigate this question in this paper for the low $\dot{M}$ optically thin regime mentioned above. In $\S 2$ we summarize the basic results of Papers 1 and 2 and write down the self-similar solutions derived in these papers. The calculations presented in later sections are based on these solutions. Then, in $\S 3$, we set up a fairly detailed set of equations to describe the heating and cooling of a two-temperature plasma in an accretion flow. We include a number of cooling processes, such as bremsstrahlung, synchrotron, and Compton cooling, and model energy transfer from the ions to the electrons through Coulomb scattering. To model these processes we make use of standard formulae taken from the literature.

We present the main results of the paper in $\S 4$. We show that the equations we develop in $\S \S 2,3$ permit three branches of solutions: the first branch is the standard Shakura-Sunyaev thin accretion disk solution, the second branch is a hot thermally unstable optically thin solution which is none other than the hot SLE solution, while the third branch is a new even hotter and more optically thin solution. The third solution is advection-dominated, as we might have suspected, and is thermally stable, again as expected. We describe the properties of the new advection-dominated solutions and determine the regions of the $\dot{M} R$ plane over which this branch of solutions is present. Some of these results were discussed by Rees et al. (1982) using a more qualitative analysis. In the calculations presented here, we model various kinds of central stars, focusing in particular on three cases, viz. a $10 M_{\odot}$ black hole, a $10^{8} M_{\odot}$ black hole, and a $1.4 M_{\odot}$ neutron star. The first and third cases are relevant to galactic X-ray binaries at low accretion rates and the second corresponds to underfed active galactic nuclei. In two recent papers, Abramowicz et al. (1995) and Chen (1995) have discussed several of the issues discussed in $\S 4$, but using a simplified singletemperature model and including only free-free cooling (see $§ 4.1$ ).

One of the results that comes out of our calculations is that for a wide range of conditions both the Shakura-Sunyaev thin disk solution and the new advection-dominated solution are present, both solutions being stable within the slim disk approximation. Which of the two solution branches will an actual accretion flow choose? We discuss this issue in $\S 5$ and make heuristic arguments to suggest that perhaps it is the advection-dominated branch which is often preferred. We conclude in $\S 6$ with a summary of the results and discuss some of the implications.

The Appendices discuss two technical points. In Appendix A, we investigate whether the non-thermal coupling of ions and electrons described by Begelman \& Chiueh (1988) might invalidate our assumption of a purely Coulomb-coupled two-temperature plasma. In Appendix B, we discuss the effect of radiative viscosity.

Readers who wish to see the main results and are not interested in the technical details of the model may wish to proceed directly to $\S 4$ at this point, or perhaps even to $\S 6$ which gives a comprehensive summary.

Before diving into the technical details in the next two sections, it is useful to mention the differences between this study and some previous works. The major feature of this work (and also the paper by Abramowicz et al. 1995) is that we systematically include entropy 
advection in the dynamics of the flow. Thereby, we are able to study optically-thin, twotemperature, advection-dominated flows, which up until now have been hardly considered at all, and if at all only in a qualitative manner. Our use of self-similar solutions (Papers 1, 2, Begelman \& Meier 1982, Spruit et al. 1987) make the dynamical structure of our accretion flows unambiguous, whereas previous studies of thick disks (e.g. Paczyński \& Wiita 1980) and ion tori (e.g. Rees et al. 1982) left some dynamical quantities unspecified. As a result, our model allows us to determine self-consistently the electron densities and temperatures, the magnetic field densities, and the resulting radiative processes. There are two technical differences between our calculations and those of SLE (see also Eardley et al. 1978). One is that by including advection we find two different hot solutions, one of which is stable, whereas without advection SLE (and later workers) could obtain only one solution, an unstable one. Secondly, most of SLE's calculations involved unsaturated Comptonization of externally supplied soft photons whereas we have an internal supply of soft photons via synchro-cyclotron emission by the thermal electrons (e.g. Rees et al. 1982). Also, we use a Comptonization formula which automatically works both in the unsaturated and saturated limits. 


\section{Advection-Dominated Accretion Flows}

\subsection{Self-Similar Advection-Dominated Flows}

In Paper 1, we derived the following self-similar solution for the radial velocity $v(R)$, angular rotation frequency $\Omega(R)$, and isothermal sound speed $c_{s}(R)$ of an advection-dominated flow (see also Spruit et al. 1987 who derived a similar solution in the context of their discussion of spiral shocks):

$$
\begin{gathered}
v(R)=-\frac{\left(5+2 \epsilon^{\prime}\right)}{3 \alpha^{2}} g\left(\alpha, \epsilon^{\prime}\right) \alpha v_{f f} \equiv-c_{1} \alpha v_{f f} \\
\Omega(R)=\left[\frac{2 \epsilon^{\prime}\left(5+2 \epsilon^{\prime}\right)}{9 \alpha^{2}} g\left(\alpha, \epsilon^{\prime}\right)\right]^{1 / 2} \frac{v_{f f}}{R} \equiv c_{2} \frac{v_{f f}}{R} \\
c_{s}^{2}(R)=\frac{2\left(5+2 \epsilon^{\prime}\right)}{9 \alpha^{2}} g\left(\alpha, \epsilon^{\prime}\right) v_{f f}^{2} \equiv c_{3} v_{f f}^{2}
\end{gathered}
$$

where

$$
\begin{gathered}
v_{f f} \equiv\left(\frac{G M}{R}\right)^{1 / 2}, \\
\epsilon^{\prime} \equiv \frac{\epsilon}{f}=\frac{1}{f}\left(\frac{5 / 3-\gamma}{\gamma-1}\right), \\
g\left(\alpha, \epsilon^{\prime}\right) \equiv\left[1+\frac{18 \alpha^{2}}{\left(5+2 \epsilon^{\prime}\right)^{2}}\right]^{1 / 2}-1 .
\end{gathered}
$$

As in Paper 1, $\alpha$ represents the standard viscosity parameter (Shakura \& Sunyaev 1973) and $\gamma$ is the ratio of specific heats. The parameter $f$, which lies in the range 0 to 1 , is the fraction of viscously dissipated energy which is advected; a fraction $1-f$ of the energy is locally radiated. The density and pressure in this solution are given by

$$
\rho=\frac{\dot{M}}{4 \pi R H|v|}, \quad \quad p=\rho c_{s}^{2},
$$

where $H$ is the vertical scale height, given approximately by

$$
H / R \approx\left(2.5 c_{3}\right)^{1 / 2}
$$

The viscous dissipation of energy per unit volume is

$$
q^{+}=\frac{3 \epsilon^{\prime} \rho|v| c_{s}^{2}}{2 R}
$$

The dissipation per unit surface area is $\mathrm{Q}^{+}=2 \mathrm{Hq}^{+}$. 
We use the above formulae in this paper to calculate the local properties of the accretion solutions. For a given choice of $\alpha, \gamma$, and $f$, these relations specify all the local properties of an advection-dominated flow (except the temperature, for which we need an equation of state). We note that, although these solutions were derived using the height-averaged slim disk equations, they are in excellent agreement with numerical solutions of a more exact set of equations which do not make the height-averaging approximation (Paper 2). Furthermore, the solutions are valid even for cooling-dominated flows with $f \rightarrow 0$; in this limit the solution reduces to a reasonable approximation of a thin accretion disk (Papers $1,2)$.

Technically, equations (2.1), (2.2) are valid only for self-similar flows extending from $R=0$ to $R=\infty$. In Paper 1 we presented some numerical solutions of the slim disk equations where we imposed boundaries at finite radii. We have also obtained other numerical solutions where we imposed a sonic boundary condition at the inner edge, appropriate to a black hole. In all cases we find that the numerical solutions quickly settle down to the self-similar form a short distance away from the boundaries. This shows that the self-similar solution is in some sense the "natural" state of the flow. Another requirement for the validity of the self-similar solution is that $f$ must be independent of $R$. But, once again, we have confirmed with numerical experiments that if $f$ varies (slowly) with $R$, then the numerical solution tracks the local self-similar form quite accurately. As a result of these and other tests we believe that eqs (2.1) and (2.2) are perfectly adequate for the calculations presented in this paper.

Finally, we mention that the solutions have been derived using a Newtonian potential. For accretion onto black holes and neutron stars, which is the primary focus of this paper, we should technically carry out the calculations in the Schwarzschild or Kerr metric. This does not appear to be a serious defect and we feel that it will introduce only small quantitative errors in some of the conclusions.

\subsection{Thermodynamics of the Gas}

We assume that the accreting gas is roughly in equipartition with an isotropically tangled magnetic field, and write the total pressure $p$ as

$$
p=p_{g}+p_{m}, \quad p_{g}=\beta p, \quad p_{m}=(1-\beta) p,
$$

where $p_{g}$ is the gas pressure, $p_{m}$ is the magnetic pressure, and we take $\beta$ to be independent of $R$. If we have flux-freezing, then we know that the magnetic field strength scales with length as $B \sim \ell^{-2}$. This means that $p_{m} \sim B^{2} \sim \rho^{4 / 3}$ and the magnetic field behaves just like radiation. Assuming such a frozen-in field, we take $\gamma$ in eq (2.2) to be given by

$$
\gamma=\Gamma_{1}=\frac{32-24 \beta-3 \beta^{2}}{24-21 \beta}
$$

where $\Gamma_{1}$ is the effective $\Gamma$ in the adiabatic $p \rho$ relation as defined by Clayton (1983). Note that the definition of $\beta$ in equation (2.6) is similar to that used in discussions of gasradiation mixtures (e.g. Clayton 1983), but differs from the usual definition of $\beta$ in the 
magnetohydrodynamics literature (the MHD $\beta$ is equal to our $\beta /(1-\beta)$ ). The internal energy per unit volume of the gas is

$$
U=\frac{3}{2} \beta p_{g}+\frac{B^{2}}{4 \pi}
$$

Ever since the important work of SLE, it has been common to discuss accretion flows in high energy astrophysics in the context of a two-temperature plasma, where the ions and the electrons have different temperatures. In keeping with this practice, we allow the ion temperature $T_{i}$ and the electron temperature $T_{e}$ to be different, and take the gas pressure of the accreting gas to be given by

$$
p_{g}=\beta \rho c_{s}^{2}=\frac{\rho k T_{i}}{\mu_{i} m_{u}}+\frac{\rho k T_{e}}{\mu_{e} m_{u}} .
$$

The effective molecular weights of the ions and electrons are given respectively by

$$
\mu_{i}=\frac{4}{1+3 X}=1.23, \quad \mu_{e}=\frac{2}{1+X}=1.14
$$

where the numerical values correspond to a hydrogen mass fraction $X=0.75$. The equipartition magnetic field is determined from the magnetic pressure:

$$
p_{m}=(1-\beta) \rho c_{s}^{2}=\frac{B^{2}}{8 \pi} \text {. }
$$

We do not include radiation pressure in our equation of state because the optically thin advection-dominated flows we consider always have gas pressure much larger than radiation pressure. If we wish to extend these studies to higher accretion rates, such as those considered by Abramowicz et al. (1988, 1995), we will need to include an additional radiation pressure term in equation (2.6).

\subsection{Scaled Numerical Relations}

We scale masses in solar units by writing

$$
M=m M_{\odot},
$$

and accretion rates in Eddington units,

$$
\dot{M}=\dot{m} \dot{M}_{E d d}, \quad \dot{M}_{E d d}=\frac{L_{E d d}}{\eta_{e f f} c^{2}}=\frac{4 \pi G M}{\eta_{e f f} \kappa_{e s} c}=1.39 \times 10^{18} \mathrm{mgs}^{-1}, \quad \eta_{e f f}=0.1
$$

where $\kappa_{e s}=0.4 \mathrm{~cm}^{2} \mathrm{~g}^{-1}$ and we have assumed the standard value for the accretion efficiency factor $\eta_{e f f}$. Also, we scale radii in Schwarzschild gravitational units,

$$
R=r R_{S c h w}, \quad R_{S c h w}=\frac{2 G M}{c^{2}}=2.95 \times 10^{5} \mathrm{~m} \mathrm{~cm} .
$$


Note that we have introduced the efficiency $\eta_{e f f}$ only for easy comparison with other works. Our accretion flows generally do not radiate with an efficiency $\eta_{e f f}$. In fact, in extremely advection-dominated flows around black holes, the actual radiation efficiency is very low because the black hole swallows all the advected energy.

With the above scalings, we can rewrite the equations of $\S \S 2.1,2.2$ as follows:

$$
\begin{gathered}
v=-2.12 \times 10^{10} \alpha c_{1} r^{-1 / 2} \mathrm{cms}^{-1}, \\
\Omega=7.19 \times 10^{4} c_{2} m^{-1} r^{-3 / 2} \mathrm{~s}^{-1}, \\
c_{s}^{2}=4.50 \times 10^{20} c_{3} r^{-1} \mathrm{~cm}^{2} \mathrm{~s}^{-2}, \\
\rho=3.79 \times 10^{-5} \alpha^{-1} c_{1}^{-1} c_{3}^{-1 / 2} m^{-1} \dot{m} r^{-3 / 2} \mathrm{gcm}^{-3}, \\
p=1.71 \times 10^{16} \alpha^{-1} c_{1}^{-1} c_{3}^{1 / 2} m^{-1} \dot{m} r^{-5 / 2} \mathrm{gcm}^{-1} \mathrm{~s}^{-2}, \\
B=6.55 \times 10^{8} \alpha^{-1 / 2}(1-\beta)^{1 / 2} c_{1}^{-1 / 2} c_{3}^{1 / 4} m^{-1 / 2} \dot{m}^{1 / 2} r^{-5 / 4} \mathrm{G}, \\
q^{+}=1.84 \times 10^{21} \epsilon^{\prime} c_{3}^{1 / 2} m^{-2} \dot{m} r^{-4} \mathrm{ergcm}^{-3} \mathrm{~s}^{-1}, \\
n_{e}=\rho / \mu_{e} m_{u}=2.00 \times 10^{19} \alpha^{-1} c_{1}^{-1} c_{3}^{-1 / 2} m^{-1} \dot{m} r^{-3 / 2} \mathrm{~cm}^{-3}, \\
\tau_{e s}=2 n_{e} \sigma_{T} H=12.4 \alpha^{-1} c_{1}^{-1} \dot{m} r^{-1 / 2} .
\end{gathered}
$$

where $n_{e}$ is the electron number density, $\tau_{e s}$ is the scattering optical depth, $\sigma_{T}=6.62 \times$ $10^{-25} \mathrm{~cm}^{2}$ is the Thomson cross-section, and the constants $c_{1}, c_{2}, c_{3}$ are defined in eq (2.1). In the case of a two-temperature plasma, equations (2.9) and (2.10) show that the ion and electron temperatures satisfy

$$
T_{i}+1.08 T_{e}=6.66 \times 10^{12} \beta c_{3} r^{-1} \mathrm{~K} .
$$




\section{Heating and Cooling of a Two-Temperature Plasma}

We determine the ion and electron temperatures in the accreting plasma by taking into account the detailed balance of heating, cooling, and advection. Due to the large mass difference between ions and electrons, we expect $q^{+}$to act primarily on the ions (e.g. SLE, Rees et al. 1982), which then transfer some of their energy to the electrons. In this paper, we assume that the transfer from ions to electrons occurs through Coulomb coupling (SLE) and we ignore other non-thermal coupling mechanisms which may be present in turbulent magnetized plasmas (e.g. Begelman \& Chiueh 1988, see Appendix A of the present paper). The cooling of the plasma is primarily via electrons and occurs through a variety of channels as we discuss below.

\subsection{Heating of Electrons by Ions}

If the ions are at a higher temperature than the electrons, Coulomb collisions transfer energy from ions to electrons at a volume transfer rate (Stepney \& Guilbert 1983)

$$
\begin{aligned}
q^{i e}= & \frac{3}{2} \\
& \frac{m_{e}}{m_{p}} n_{e} n_{i} \sigma_{T} c \frac{\left(k T_{i}-k T_{e}\right)}{K_{2}\left(1 / \theta_{e}\right) K_{2}\left(1 / \theta_{i}\right)} \ln \Lambda \\
& \times\left[\frac{2\left(\theta_{e}+\theta_{i}\right)^{2}+1}{\left(\theta_{e}+\theta_{i}\right)} K_{1}\left(\frac{\theta_{e}+\theta_{i}}{\theta_{e} \theta_{i}}\right)+2 K_{0}\left(\frac{\theta_{e}+\theta_{i}}{\theta_{e} \theta_{i}}\right)\right] \mathrm{ergcm}^{-3} \mathrm{~s}^{-1},
\end{aligned}
$$

where the $K$ 's are modified Bessel functions, the Coulomb logarithm is roughly $\ln \Lambda=20$, and the dimensionless electron and ion temperatures are defined by

$$
\theta_{e}=\frac{k T_{e}}{m_{e} c^{2}}, \quad \theta_{i}=\frac{k T_{i}}{m_{p} c^{2}}
$$

Equation (3.1) assumes that all the ions are protons. For a more general case, we should replace $n_{i}$ by a sum over ion species, $\sum Z_{j}^{2} n_{j}$, where $Z_{j}$ is the charge of the $j$-th species and $n_{j}$ is its number density. This gives a factor of 1.25 for a composition of $75 \% \mathrm{H}$ and $25 \%$ He. Thus

$$
\begin{aligned}
q^{i e}= & 5.61 \times 10^{-32} \frac{n_{e} n_{i}\left(T_{i}-T_{e}\right)}{K_{2}\left(1 / \theta_{e}\right) K_{2}\left(1 / \theta_{i}\right)} \\
& \times\left[\frac{2\left(\theta_{e}+\theta_{i}\right)^{2}+1}{\left(\theta_{e}+\theta_{i}\right)} K_{1}\left(\frac{\theta_{e}+\theta_{i}}{\theta_{e} \theta_{i}}\right)+2 K_{0}\left(\frac{\theta_{e}+\theta_{i}}{\theta_{e} \theta_{i}}\right)\right] \mathrm{ergcm}^{-3} \mathrm{~s}^{-1} .
\end{aligned}
$$

\subsection{Cooling of Electrons}

Electrons are cooled by many different processes including bremsstrahlung, synchrocyclotron, and Compton cooling off soft photons. 


\section{Bremsstrahlung Cooling}

The electrons cool both by electron-ion and electron-electron bremsstrahlung. We write the cooling rate per unit volume as

$$
q_{b r}^{-}=q_{e i}^{-}+q_{e e}^{-},
$$

where the subscripts $e i$ and $e e$ denote the electron-ion and the electron-electron rates. Following Stepney and Guilbert (1983), we adopt

$$
q_{e i}^{-}=1.25 n_{e}^{2} \sigma_{T} c \alpha_{f} m_{e} c^{2} F_{e i}\left(\theta_{e}\right)=1.48 \times 10^{-22} n_{e}^{2} F_{e i}\left(\theta_{e}\right) \mathrm{ergcm}^{-3} \mathrm{~s}^{-1},
$$

where $\alpha_{f}$ is the fine structure constant and we have included a factor of 1.25 for the same reason as in eq (3.3). The function $F_{e i}\left(\theta_{e}\right)$ has the approximate form

$$
\begin{aligned}
F_{e i}\left(\theta_{e}\right) & =4\left(\frac{2 \theta_{e}}{\pi^{3}}\right)^{1 / 2}\left[1+1.781 \theta_{e}^{1.34}\right], & & \theta_{e}<1, \\
& =\frac{9 \theta_{e}}{2 \pi}\left[\ln \left(1.123 \theta_{e}+0.48\right)+1.5\right], & & \theta_{e}>1 .
\end{aligned}
$$

The numerical constant 0.48 in the second expression is quoted as 0.42 by Stepney \& Guilbert (1983) but we have changed the value in order to have a continuous $F_{e i}\left(\theta_{e}\right)$ across $\theta_{e}=1$. For the electron-electron bremsstrahlung, Svensson (1982) gives expressions in the limits $\theta_{e}<1$ and $\theta_{e}>1$. For $\theta_{e}<1$,

$$
\begin{aligned}
q_{e e}^{-} & =n_{e}^{2} c r_{e}^{2} m_{e} c^{2} \alpha_{f} \frac{20}{9 \pi^{1 / 2}}\left(44-3 \pi^{2}\right) \theta_{e}^{3 / 2}\left(1+1.1 \theta_{e}+\theta_{e}^{2}-1.25 \theta_{e}^{5 / 2}\right) \\
& =2.56 \times 10^{-22} n_{e}^{2} \theta_{e}^{3 / 2}\left(1+1.1 \theta_{e}+\theta_{e}^{2}-1.25 \theta_{e}^{5 / 2}\right) \mathrm{ergcm}^{-3} \mathrm{~s}^{-1}
\end{aligned}
$$

while for $\theta_{e}>1$,

$$
\begin{aligned}
q_{e e}^{-} & =n_{e}^{2} c_{e}^{2} m_{e} c^{2} \alpha_{f} 24 \theta_{e}\left(\ln 2 \eta \theta_{e}+1.28\right) \\
& =3.40 \times 10^{-22} n_{e}^{2} \theta_{e}\left(\ln 1.123 \theta_{e}+1.28\right) \mathrm{ergcm}^{-3} \mathrm{~s}^{-1},
\end{aligned}
$$

where $r_{e}=e^{2} / m_{e} c^{2}$ is the classical electron radius and $\eta=\exp \left(-\gamma_{E}\right)=0.5616$. For $\theta_{e}>1$, Svensson (1982) gives a numerical constant of $5 / 4$ instead of 1.28 inside the parentheses; again we have changed the constant to ensure smoothness across $\theta_{e}=1$.

\section{Synchrotron Cooling}

Cooling via synchrotron radiation is not often considered in thermal models of X-ray binaries (e.g. SLE and Wandel \& Liang 1991 ignore it but Kusunose \& Takahara 1989 do include it), though it is fairly common in discussions of AGN models (e.g. the ion torus model of Rees et al. 1982). Due to the assumption of an equipartition magnetic field in the 
plasma, synchrotron emission from thermal electrons can be quite important in our model, and we therefore include it in the calculations.

In the optically thin limit, the spectrum of synchrotron emission by a relativistic Maxwellian distribution of electrons is given by Pacholczyk (1970),

$$
\epsilon_{\text {synch }} d \nu=4.43 \times 10^{-30} \frac{n_{e} \nu}{K_{2}\left(1 / \theta_{e}\right)} I\left(\frac{x_{M}}{\sin \theta}\right) d \nu \operatorname{ergcm}^{-3} \mathrm{~s}^{-1} \mathrm{~Hz}^{-1},
$$

where $I(x)$ is a tabulated function,

$$
x_{M} \equiv \frac{2 \nu}{3 \nu_{0} \theta_{e}^{2}}, \quad \nu_{0} \equiv \frac{e B}{2 \pi m_{e} c}
$$

and $\theta$ is the angle between the velocity vector of the electron and the direction of the local magnetic field. Mahadevan, Narayan \& Yi (1995) have obtained an extremely accurate fitting function (maximum error 0.0036) for $I\left(x_{M}\right)$,

$$
I\left(x_{M}\right)=2.5651\left(1+\frac{1.92}{x_{M}^{1 / 3}}+\frac{0.9977}{x_{M}^{2 / 3}}\right) \exp \left(-1.8899 x_{M}^{1 / 3}\right)
$$

Averaging over $\theta$ for an isotropic velocity distribution, $I\left(x_{M} / \sin \theta\right)$ gets replaced by a new function $I^{\prime}\left(x_{M}\right)$, for which the fitting function (max error 0.015 ) is

$$
I^{\prime}\left(x_{M}\right)=\frac{4.0505}{x_{M}^{1 / 6}}\left(1+\frac{0.40}{x_{M}^{1 / 4}}+\frac{0.5316}{x_{M}^{1 / 2}}\right) \exp \left(-1.8899 x_{M}^{1 / 3}\right) .
$$

Although these formulae are valid only for relativistic temperatures, $\theta_{e} \gtrsim 1$, we use them at all temperatures. In our models, the synchrotron emission always comes from relativistic electrons in the tail of the Maxwellian distribution, even if the temperature is somewhat sub-relativistic, and so this approximation is reasonable.

Optically thin synchrotron emission rises steeply with decreasing frequency $\nu$. Under most circumstances, the emission is self-absorbed below a critical frequency $\nu_{c}$. We estimate $\nu_{c}$ by the following approximate calculation. At radius $\mathrm{R}$, we assume that the synchrotron emission occurs over the volume of a sphere of radius $\mathrm{R}$, and we equate this emission to the Rayleigh-Jeans blackbody emission from the surface of the sphere. This gives the condition

$$
4.43 \times 10^{-30} \frac{n_{e} \nu}{K_{2}\left(1 / \theta_{e}\right)} I^{\prime}\left(x_{M}\right) \cdot \frac{4 \pi}{3} R^{3}=\pi \cdot 2 \frac{\nu^{2}}{c^{2}} k T_{e} \cdot 4 \pi R^{2} .
$$

Substituting for $I^{\prime}\left(x_{M}\right)$, we then obtain a transcendental equation for $x_{M}$,

$$
\exp \left(1.8899 x_{M}^{1 / 3}\right)=2.49 \times 10^{-10} \frac{n_{e} R}{B} \frac{1}{\theta_{e}^{3} K_{2}\left(1 / \theta_{e}\right)}\left(\frac{1}{x_{M}^{7 / 6}}+\frac{0.40}{x_{M}^{17 / 12}}+\frac{0.5316}{x_{M}^{5 / 3}}\right)
$$


We solve this equation numerically at each $R$ to obtain $x_{M}$, and thereby calculate the critical frequency $\nu_{c}$ by means of the relation

$$
\nu_{c}=\frac{3}{2} \nu_{0} \theta_{e}^{2} x_{M}, \quad \nu_{0}=2.80 \times 10^{6} \mathrm{BHz}
$$

where $B$ is expressed in Gauss. Technically, the above calculation is valid only for a spherical flow and we need to introduce corrections of order $H / R$ for a rotationally flattened flow. However, synchrotron emission is important only in the hot highly advection-dominated solutions and these are essentially spherical (Paper 2). We therefore feel these formulae are adequate.

To estimate the cooling per unit volume due to synchrotron radiation we use the relation

$$
q_{\text {synch }}^{-} \cdot 4 \pi R^{2} \Delta R=\pi \cdot 2 \frac{\nu_{c}^{2}(R)}{c^{2}} k T_{e}(R) \frac{d \nu_{c}(R)}{d R} \Delta R \cdot 4 \pi R^{2}
$$

The left hand side of this equation is the total cooling over a shell extending from radius $R$ to $R+d R$. The right-hand side represents the net flux reaching an observer at infinity from this shell. This expression is obtained by assuming that at each frequency $\nu$ the observer sees a blackbody source with a radius determined by the condition $\nu=\nu_{c}(R)$. We thus obtain the cooling rate per unit volume to be

$$
q_{\text {synch }}^{-}=\frac{2 \pi}{3 c^{2}} k T_{e}(R) \frac{d \nu_{c}^{3}(R)}{d R}
$$

This result is valid only if $\nu_{c}$ increases monotonically with decreasing $R$, which is satisfied by all our models. Equation (3.17) is a non-local expression for the synchrotron emissivity since it depends on properties at neighboring $R$. For even greater simplicity, we often adopt a purely "local" approximation, in which the derivative of $\nu_{c}^{3}$ with respect to $R$ is simply replaced by $\nu_{c}^{3} / R$. Thus we have approximately

$$
q_{\text {synch }}^{-} \approx \frac{2 \pi}{3 c^{2}} k T_{e}(R) \frac{\nu_{c}^{3}(R)}{R} .
$$

Note that the simple treatment of synchrotron emission discussed here ignores energy transport from one radius to another by radiative diffusion of the self-absorbed photons. It is not clear how large this effect is. Note also that the cooling rate given here is different from other expressions given in the literature (e.g. Ipser \& Price 1983). Equation (3.17) has been written in a form that explicitly ensures that the integral of $q_{\text {synch }}^{-}$over the entire flow is equal to the total cooling radiation that reaches infinity.

\section{Compton Cooling}

Although we neglect nonlocal radiative transfer effects in the optically thin situations considered in this paper, we cannot afford to ignore the scattering of escaping photons off hot thermal electrons. Indeed, in some of our models the scattering depth is comparable to unity and Comptonization of soft photons by hot electrons becomes an important cooling mechanism, especially in the inner regions of the flow. 
Dermer, Liang \& Canfield (1991) have given a convenient though approximate prescription for the Comptonized energy enhancement factor $\eta$, which is defined to be the average change in energy of a photon between injection and escape. Their prescription is

$$
\eta=1+\frac{P(A-1)}{(1-P A)}\left[1-\left(\frac{x}{3 \theta_{e}}\right)^{-1-\ln P / \ln A}\right] \equiv 1+\eta_{1}-\eta_{2}\left(\frac{x}{\theta_{e}}\right)^{\eta_{3}},
$$

where

$$
\begin{aligned}
x & =h \nu / m_{e} c^{2}, \\
P & =1-\exp \left(-\tau_{e s}\right), \\
A & =1+4 \theta_{e}+16 \theta_{e}^{2} .
\end{aligned}
$$

The factor $P$ is the probability that an escaping photon is scattered, while $A$ is the mean amplification factor in the energy of a scattered photon when the scattering electrons have a Maxwellian velocity distribution of temperature $\theta_{e}$. Note that the extra cooling due to Comptonization is $\eta-1$ times the mean flux of escaping photons. The formula (3.19) is valid only for soft photons, i.e. for $x<3 \theta_{e}$. Hard photons actually heat up the electrons, but we ignore this since our models generally have very little hard radiation capable of causing Compton-heating.

An important feature of the Dermer et al. formula is that it treats both the unsaturated and saturated limits of Comptonization. SLE considered only the unsaturated Comptonization limit where the Compton $y$-parameter is taken to be around unity. They also assumed an external supply of soft photons and solved the steady-state Kompaneets equation to obtain the spectrum (cf. Eardley et al. 1978). The formulae given above use the Thomson cross-section, whereas one should technically employ the Klein-Nishina cross-section at these temperatures. In practice, this simplification is not serious. The bulk of the Comptonization occurs on very soft photons, either synchrotron radiation or thermal radiation from the accreting star, and these photons remain soft even in the rest frame of the electrons. Therefore, for single or even double Compton scattering the Thomson approximation is very good. Saturated Comptonization occurs only for limiting solutions, near the critical $\dot{m}_{\text {crit }}$ lines discussed in $\S 4$; in these solutions the electron temperature tends to be a little lower, $T_{e} \lesssim 10^{9.5} \mathrm{~K}$, where the Klein-Nishina correction is less important.

In our models, Comptonization can operate on three different sources of radiation, and we need to calculate the cooling due to each of these.

Comptonization of Bremsstrahlung Radiation: We have earlier estimated the total cooling $q_{b r}^{-}$due to bremsstrahlung. The spectrum of the emission per unit volume may be written as a function of $x=h \nu / m_{e} c^{2}$ as follows:

$$
\epsilon_{b r}\left(\frac{x}{\theta_{e}}\right) d\left(\frac{x}{\theta_{e}}\right)=q_{b r}^{-} \exp \left(-\frac{x}{\theta_{e}}\right) d\left(\frac{x}{\theta_{e}}\right) .
$$

This spectrum, which corresponds to the electron-ion emission, may not adequately describe electron-electron bremsstrahlung, but the latter is not important at the temperatures we are 
interested in. For computational convenience, we follow Dermer et al. (1991) and replace eq (3.20a) by the following simple function,

$$
\begin{aligned}
\epsilon_{b r}\left(\frac{x}{\theta_{e}}\right) d\left(\frac{x}{\theta_{e}}\right) & =q_{b r}^{-} d\left(\frac{x}{\theta_{e}}\right), & & \frac{x}{\theta_{e}} \leq 1 \\
& =0, & & \frac{x}{\theta_{e}}>1 .
\end{aligned}
$$

The part of the spectrum which can be Comptonized is the range from $x=x_{c}=h \nu_{c} / m_{e} c^{2}$ at the synchrotron self-absorption edge upto $x=\theta_{e}$. Thus, the volume rate of cooling of electrons by self-Comptonization of bremsstrahlung radiation is

$$
q_{b r, C}^{-}=3 \eta_{1} q_{b r}^{-}\left[\left(\frac{1}{3}-\frac{x_{c}}{3 \theta_{e}}\right)-\frac{1}{\eta_{3}+1}\left(\left(\frac{1}{3}\right)^{\eta_{3}+1}-\left(\frac{1}{3 \theta_{e}}\right)^{\eta_{3}+1}\right)\right] .
$$

Comptonization of Synchrotron Radiation: In our simple model of synchrotron cooling, the escaping radiation is emitted mostly at the local self-absorption cut-off frequency $\nu_{c}$ given by equation (3.15). The Comptonization of this radiation gives an additional cooling rate

$$
q_{\text {synch }, C}^{-}=q_{\text {synch }}^{-}\left[\eta_{1}-\eta_{2}\left(\frac{x_{c}}{\theta_{e}}\right)^{\eta_{3}}\right] \text {. }
$$

Comptonization of Soft photons from the Star: A third source of soft photons is radiation emitted by the accreting star. SLE were the first to note that Comptonization of externally supplied soft photons can be a very efficient cooling process. The soft photons may arise from a standard thin accretion disk, or from the companion star in a binary. We consider here soft photons which may be emitted by the accreting star. In principle, the star may have its own internal source of luminosity. However, we assume that the dominant stellar luminosity is that which arises from the accretion itself, viz. the internal energy of the hot accreting material which is thermalized and radiated at the stellar surface. The luminosity due to this component is

$$
L_{*}=4 \pi R_{*} H\left(R_{*}\right)\left|v\left(R_{*}\right)\right|\left[U\left(R_{*}\right)+\frac{1}{2} \rho\left(R_{*}\right) v^{2}\left(R_{*}\right)\right]
$$

where $R_{*}$ is the radius of the star and $U$ is the internal energy of the accreting gas (eq 2.11). In eq (3.25) we have included the radial kinetic energy of the gas in calculating $L_{*}$ but not the rotational energy. This is because we assume that the star is spinning in equilibrium with the accreting material.

It is unclear at this time exactly what the spectrum of the re-emitted radiation will be. One possibility is that all the incoming internal energy in the accretion flow is fully thermalized at the stellar surface so that the star radiates essentially as a blackbody. In that case, the temperature of the radiation from the star is given by

$$
T_{*}=\left(\frac{L_{*}}{4 \pi R_{*}^{2} \sigma}\right)^{1 / 4}
$$


However, it is quite possible that thermalization is incomplete and that the radiation comes out with a much harder spectrum than a blackbody at $T_{*}$. The outgoing flux at radius $R$ is given by

$$
F_{*}(R)=\frac{L_{*}}{4 \pi R^{2}} .
$$

If the stellar radiation is blackbody, then in the same spirit as eq (3.22), we can approximate the spectrum as a pure Rayleigh-Jeans $\nu^{2}$ spectrum with a sharp cut-off at $\nu_{b}$, where $\nu_{b}$ is given by

$$
\sigma T_{*}^{4}=\pi \int_{0}^{\nu_{b}} 2 \frac{\nu^{2}}{c^{2}} k T_{*} d \nu \quad \rightarrow \quad \nu_{b}=5.61 \times 10^{10} T_{*} \mathrm{~s}^{-1} .
$$

We thus write the spectrum approximately as

$$
\begin{array}{rlrl}
F\left(\frac{x}{\theta_{e}}\right) d\left(\frac{x}{\theta_{e}}\right) & =3 F_{*}\left(\frac{\theta_{e}}{x_{b}}\right)^{3}\left(\frac{x}{\theta_{e}}\right)^{2} d\left(\frac{x}{\theta_{e}}\right), & & x \leq x_{b}=\frac{h \nu_{b}}{m_{e} c^{2}}, \\
& =0, & x>x_{b} .
\end{array}
$$

The part of the flux between $x=x_{c}$ and $x=x_{b}$ will be Comptonized provided $x_{b}<3 \theta_{e}$ (Wien limit). If $x_{b}>3 \theta_{e}$, then only the part from $x=x_{c}$ to $x=3 \theta_{e}$ is Comptonized. We ignore Compton heating.

We can calculate the Comptonization of the stellar flux just as we did the bremsstrahlung case, except that we must replace $\epsilon_{b r}$ by $F / R$. Thus, we obtain a cooling rate

$$
q_{*, C}^{-}=3 \frac{F_{*}}{R}\left(\frac{\theta_{e}}{x_{b}}\right)^{3}\left[\frac{\eta_{1}}{3}\left\{\left(\frac{x_{\max }}{\theta_{e}}\right)^{3}-\left(\frac{x_{c}}{\theta_{e}}\right)^{3}\right\}-\frac{\eta_{2}}{3+\eta_{3}}\left\{\left(\frac{x_{\max }}{\theta_{e}}\right)^{3+\eta_{3}}-\left(\frac{x_{c}}{\theta_{e}}\right)^{3+\eta_{3}}\right\}\right],
$$

where $x_{\max }=\max \left(x_{b}, 3 \theta_{e}\right)$. Note that the cooling term $q_{*, C}^{-}$is included only in the case of accretion on a normal star such as a neutron star. We do not include this term for accretion on a black hole which lacks a radiating surface. Note also that the analysis we have presented is valid only when the accreting gas is optically very thin. In some of the solutions described in this paper the optical depth approaches unity and we expect substantial radiative transfer effects in the propagation of the soft photons from the star. It is beyond the scope of this paper to model these effects accurately.

\section{Optically Thick Cooling}

For the advection-dominated flows that we concentrate on in this paper, it is sufficient to set the volume rate of cooling equal to the sum of the five cooling rates given above, viz. $q_{b r}^{-}, q_{s y n c h}^{-}, q_{b r, C}^{-}, q_{s y n c h, C}^{-}, q_{*, C}^{-}$. However, we also calculate solutions corresponding to normal thin accretion disks. These solutions are optically thick and radiate like a blackbody or a modified blackbody. Since this is a very different regime, we need to generalize our cooling formula.

Hubeny (1990) has shown that the effective surface flux $F_{v}$ from an accretion disk can be written approximately as

$$
F_{v}=\sigma T_{e f f}^{4}=\frac{4 \sigma T_{e}^{4}}{\frac{3 \tau}{2}+\sqrt{3}+\frac{1}{\tau_{a b s}}}
$$


where $\tau=\tau_{a b s}+\tau_{e s}$ is the total optical depth in the vertical direction from the disk midplane to the surface, $\tau_{a b s}$ is the corresponding absorption optical depth, $T_{e f f}$ is the effective surface temperature, and $T_{e}$ is the electron temperature at the equatorial plane. Equation (2.15) gives an expression for $\tau_{e s}$, but to calculate $\tau_{a b s}$ we need to evaluate all the relevant emission and absorption processes. Note however that, in the extreme optically thin limit, eq (3.31) gives $F_{v}=4 \sigma T_{e}^{4} \tau_{a b s}$. Since the previous sections give the net cooling in precisely this limit, we can therefore estimate $\tau_{a b s}$ approximately by

$$
\tau_{a b s}=\frac{H}{4 \sigma T_{e}^{4}}\left(q_{b r}^{-}+q_{s y n c h}^{-}+q_{b r, C}^{-}+q_{s y n c h, C}^{-}+q_{*, C}^{-}\right)
$$

Substituting this estimate of $\tau_{a b s}$ back into eq (3.31), we obtain a modified expression for the net volume cooling rate of the accreting gas:

$$
q^{-}=\frac{F_{v}}{H}=\frac{4 \sigma T_{e}^{4} / H}{\frac{3 \tau}{2}+\sqrt{3}+\frac{4 \sigma T_{e}^{4}}{H}\left(q_{b r}^{-}+q_{s y n c h}^{-}+q_{b r, C}^{-}+q_{s y n c h, C}^{-}+q_{*, C}^{-}\right)^{-1}} .
$$

Equation (3.33) is valid both in the optically thick and thin limits. When the gas is extremely optically thick, this formula gives $q^{-}=8 \sigma T_{e}^{4} / 3 H \tau$ which is the appropriate blackbody limit, whereas in the optically thin limit it gives $q^{-}=q_{b r}^{-}+q_{\text {synch }}^{-}+q_{b r, C}^{-}+q_{\text {synch,C }}^{-}+q_{*, C}^{-}$. The formula thus provides a convenient interpolation between the two limits.

\subsection{Thermal Balance Equations}

Using the heating and cooling rates given above, we are in a position to solve for the energy balance of the accreting medium. In our model, a fraction $f$ of the viscously dissipated energy $q^{+}$is advected inward while a fraction $1-f$ is transferred from ions to electrons and then radiated. We therefore have two relations which finally close the set of equations and help determine the ion and electron temperatures.

Consider first the energy balance of the ions. We require the rate of input of energy through viscous dissipation to be equal to the sum of the rate of advection of energy by the ions and the rate of transfer of energy from the ions to the electrons. This gives the condition

$$
q^{+}=q^{a d v}+q^{i e}=f q^{+}+q^{i e},
$$

where $q^{+}$and $q^{i e}$ are given in eqs (2.5) and (3.3), and we set $q^{a d v}=f q^{+}$by the definition of $f$. In writing this equation we have assumed that all the viscous energy goes into the ions, which is reasonable since the ions are very much more massive than the electrons.

We obtain a second equation by considering the energy balance of the electrons. In this case we require the net heating and cooling rates of the electrons to be equal, which gives

$$
q^{i e}=q^{-}
$$

where $q^{-}$is calculated via eq (3.33).

For a given $M, \dot{M}, R, \alpha, \beta$, we can think of equations (2.16), (3.34) and (3.35) as three relations which may be solved to obtain the ion and electron temperatures, $T_{i}, T_{e}$, and the advective fraction $f$. The other parameters of the gas such as $v, \rho, B$, etc., which are needed 
to calculate $q^{+}, q^{i e}$ and $q^{-}$, are given in eq (2.15). Since the equations are fairly complex we solve them numerically. The important point however is that the solutions we obtain are fully self-consistent within the framework of our model. Indeed, considering that we have included advection, and that we model a two-temperature plasma along with a variety of cooling mechanisms including synchrotron emission and Comptonization with proper treatment of saturation, the calculations presented here may well be the most complete and self-consistent computations attempted of a high energy accretion flow.

A point worth emphasizing is the presence of the advection term $q^{a d v}$ in equation (3.34). It is this term above all else, coupled with the use of the self-similar solution discussed in $\S 2$, which sets this work apart from earlier analyses of accretion flows. In virtually all previous studies it has been the practice to assume local energy balance. This means that in single temperature models one requires $q^{+}=q^{-}$, while in two-temperature models one tries to satisfy $q^{+}=q^{i e}=q^{-}$. Our solutions are more general because they allow for advection. Moreover, the fraction $f$ of the energy which is advected is not assigned arbitrarily but is solved for self-consistently, as are the ion and electron temperatures and other properties of the accreting gas. Because of this we are able to reproduce the two previously known solutions, namely the Shakura-Sunyaev thin disk and the SLE hot disk, both of which have negligible advection $(f \ll 1)$, and in addition we obtain a new class of very hot solutions which are advection-dominated $(f \rightarrow 1)$. 


\section{Results}

As we have discussed in $\S 1$ (see also Papers 1,2), advection-dominated accretion can arise both in the optically thin and optically thick limits. In this paper we restrict our investigation to the optically thin case. The paper by Rees et al. (1982) on ion tori showed that there is an upper limit to the mass accretion rate such that only below this limit is optically thin advection-dominated accretion possible. Above the limiting $\dot{M}$, the cooling is too efficient, and the only configuration allowed for the flow is the standard thin accretion disk. We explore this point here quantitatively using the detailed formalism described in the previous two sections.

\subsection{Critical Accretion Rate for a Special Case}

We begin by discussing a particularly simple case where the accreting material is cool and single-temperature, and the dominant cooling process is bremsstrahlung. This case has been considered by Abramowicz et al. (1995) and Chen (1995) who derived a formula for the maximum $\dot{M}$ up to which an advection-dominated solution is possible. To derive the formula, we choose a value for the parameter $f$, the fraction of the dissipated energy which is advected with the flow. We then equate the local bremsstrahlung cooling rate, $q_{b r}^{-}$, to a fraction $1-f$ of the heating rate $q^{+}$(see equation 3.34). Setting $T_{i}=T_{e}$ in eq (2.16) and using the non-relativistic limit of eqs. (3.6) and (3.7), this calculation gives for the scaled accretion rate (see eqs $2.12-2.14$ for the scalings)

$$
\dot{m}=1.32 \times 10^{3}(1-f) \epsilon^{\prime} \alpha^{2} \beta^{-1 / 2} c_{1}^{2} c_{3} r^{-1 / 2} .
$$

Let us set $f=1 / 2$ in this equation. This corresponds to half the released energy being advected and half being radiated, which would appear to be a reasonable choice for this calculation. Assuming $\alpha=0.3, \beta=0.5$ as a specific example, eq (4.1) gives $\dot{m}=4.5 r^{-1 / 2}$ for the limiting accretion rate up to which an optically thin advection-dominated solution is possible. This result is roughly similar to that obtained by Abramowicz et al. (1995) and Chen (1995). We see that the critical $\dot{m}$ when expressed in scaled units does not depend explicitly on the mass of the accreting star.

We can compare the analytical result (4.1) with more elaborate numerical results obtained by solving the equations written down in $\S \S 2,3$. Figure 1 shows the numerically calculated $\dot{m}$ as a function of scaled radius $r$ for a $10 M_{\odot}$ black hole. The calculation assumes $\alpha=0.3, \beta=0.5, f=0.5$ as before and the exact results are compared with the analytical formula (4.1). We see that the formula (4.1) is in excellent agreement with the numerical results at large radii, $r \geq 10^{3}$, where the plasma is single-temperature and the cooling is indeed dominated by bremsstrahlung as assumed. However, there are significant differences for $1 \leq r \leq 10^{3}$. This is because the plasma here is much hotter, the ion-electron coupling is weaker, and cooling processes are much more complex. Therefore, it is necessary to allow for a two-temperature plasma and to keep all terms in the energy balance equations.

\subsection{Three Solution Branches}

Before we proceed further, we briefly discuss the relation between the advection-dominated flows which we focus on in this paper and the standard cooling-dominated flows which 
are generally discussed in the literature. Formally, in our models, the parameter $f$ distinguishes between the two types of flow; advection-dominated flows have $f \sim 1$ while cooling-dominated flows have $f \ll 1$. We show now that, under a given set of conditions, one or the other, or possibly both, of these kinds of solutions are allowed for an accretion flow.

As an example, we consider the case of an accreting $10 M_{\odot}$ black hole with $\alpha=0.3$, $\beta=0.5$, and focus on some particular radius, say $r=10^{3}$. Given these parameters, our model allows us to solve for $f$ as a function of $\dot{m}$, or vice versa; that is, the equations written down in $\S \S 2,3$ define a unique mapping between $f$ and $\dot{m}$. We show in Fig. 2 the nature of the mapping for the case under consideration. Both at very low $\dot{m}$ and very high $\dot{m}$ there is only one solution allowed for $f$ and therefore there is only one kind of flow allowed. However, for intermediate values of $\dot{m}$ we see that there are three separate solutions. The uppermost branch, with a large value of $f$, is the advection-dominated branch which we study in this paper, and which has been discovered independently by Abramowicz et al. (1995). The lowermost branch has a very low value of $f$ and is therefore dominated by cooling. This solution has a low temperature, is optically thick, and corresponds to the well-known standard thin accretion disk solution (e.g. Shakura \& Sunyaev 1973, Frank et al. 1992). Both of these branches are stable at this radius. In addition, there is a middle branch, which is thermally unstable (see $\S 4.7$ ), and which we indicate by a dotted line. This solution has a low value of $f \ll 1$ and is therefore cooling-dominated (though its value of $f$ is larger than that of the Shakura-Sunyaev solution). This solution is much hotter than the thin disk branch and is, in fact, the unstable SLE hot solution.

The upper branch in Fig. 2 does not extend above a certain maximum accretion rate $\dot{m}_{\text {crit }}$. In this particular example corresponding to $r=10^{3}$, the critical configuration corresponds to $\dot{m}_{\text {crit }}=0.16, f=0.27$. Note that in the simplified discussion of $\S 4.1$ we arbitrarily set $f=0.5$ in order to derive eq (4.1) and also to calculate the numerical line shown in Fig. 1. The correct procedure is to vary $f$ and to optimize it so as to determine the true maximum or critical accretion rate. We employ this procedure to derive all the results presented in the sections below.

We see from Fig. 2 that there is a second critical accretion rate, a minimum $\dot{m}$ below which the standard cooling-dominated branch ceases to exist. This limit, which we designate $\dot{m}_{\text {crit }}^{\prime}$, corresponds to the point where the optical depth $\tau$ of a thin accretion disk becomes equal to unity

$$
\dot{m}=2.6 \times 10^{-8} \alpha^{4} \approx \dot{m}_{c r i t}^{\prime} .
$$

Below this accretion rate, the optical depth falls below unity and the solution goes unstable by the classic thermal instability associated with optically thin bremsstrahlung cooling (Pringle et al. 1973, Piran 1978). The second critical rate $\dot{m}_{c r i t}^{\prime}$ corresponds to this instability. Setting $\alpha=0.3$ eq (4.2) gives $\dot{m} \sim 10^{-10}$. The actual limiting $\dot{m}$ where the cooling branch terminates in Fig. 2 is a couple of orders lower because of differences in the numerical factors. We should warn the reader that the opacities we have used are not reliable at the low temperatures at which this limit occurs, so that the results are not accurate. However, the existence of the second critical $\dot{m}_{c r i t}^{\prime}$ is valid regardless of the details of the opacities, so that the overall topology of Fig. 2 is correct.

\subsection{Stellar Mass Black Holes}


Since a black hole lacks a hard radiating surface, energy which is advected with the accretion flow is lost into the black hole without being reprocessed and radiated. This is very different from the case of a neutron star where all the advected energy must ultimately be radiated from the stellar surface. This difference could potentially lead to observable signatures which may distinguish black holes from neutron stars (cf. Sunyaev \& Titarchuk 1980, Sunyaev et al. 1991ab, Liang 1993). In this and the next subsection we discuss accreting black holes, and in $\S 4.5$ we discuss accreting neutron stars.

Figure 3 shows some results corresponding to advection-dominated accretion on a $10 M_{\odot}$ black hole with $\beta=0.5$, where we remind the reader that the fraction of the total pressure due to magnetic fields is $(1-\beta)$. The solid lines in the upper panels indicate the critical accretion rate $\dot{m}_{c r i t}$ as a function of the radius $r$ for three choices of $\alpha: 0.03,0.1,0.3$. The procedure we employ to calculate the critical lines is to optimize at each $r$ the value of $f$ so as to find the maximum $\dot{m}$ for which the advection-dominated branch exists at that $r$. The typical value of $f$ on the lines is $\sim 0.3$. We see from Fig. 3 that $\dot{m}_{c r i t}$ depends sensitively on $\alpha$. For a large $\alpha=0.3$, for instance, advection-dominated accretion occurs for $\dot{m}$ as high as $\dot{m} \sim 0.1$ while for $\alpha=0.03$ we need $\dot{m}<0.001$. The values of $\dot{m}_{\text {crit }}$ scale essentially as $\alpha^{2}$, as shown by equation (4.1) (see also Rees et al. 1982). In contrast to the $\alpha$ dependence, we find that the solutions are fairly insensitive to the value of $\beta$. For instance, if we change $\beta$ from 0.5 (gas pressure $=$ magnetic pressure) to 0.9 (gas pressure $=90 \%$ of total pressure), $\dot{m}_{\text {crit }}$ moves by only $\sim 10 \%$. To indicate how the advection-dominated solutions evolve as we move down from the critical line we show in Fig. 3 a second set of lines corresponding to $f=0.9$. We see that the degree of advection-domination increases quite rapidly with decreasing $\dot{m}$. Indeed, for $\dot{m} \ll \dot{m}_{\text {crit }}$, the solutions have $f$ practically equal to unity so that there is virtually no cooling at all.

The lower panels in Fig. 3 show the ion and electron temperatures as a function of $r$ as we move along the lines corresponding to $\dot{m}=\dot{m}_{\text {crit }}$ and $f=0.9$ in the upper panels. We see that the flow has two very distinct zones. For $r \gtrsim 10^{3}$, the ions and electrons have almost the same temperature, both being nearly at the virial temperature. This is the region where the ions and electrons are well coupled and bremsstrahlung cooling dominates, so that the simple expression given in eq (4.1) is valid (see Fig. 1, and also Abramowicz et al. 1995, Chen 1995). However, for $r<10^{3}$, the temperature becomes very high and the ionelectron coupling becomes weak. Other cooling processes also enter the picture. Synchrotron emission from thermal electrons dominates over bremsstrahlung, and also Comptonization of the synchrotron and bremsstrahlung photons becomes important. As a consequence of these new effects the plasma switches to a two-temperature state. The ion temperature continues to track the virial temperature and rises monotonically inwards. However, the electron temperature saturates at a few $\times 10^{9} \mathrm{~K}$ and remains nearly constant all the way down to the Schwarzschild radius. The $f=0.9$ curves show that with decreasing $\dot{m}$, i.e. increasing $f$, the electron temperature rises in the inner region. This is because the density decreases and some of the cooling processes are less efficient. Indeed, for highly advectiondominated flows with $\dot{m} \ll \dot{m}_{\text {crit }}$, the electron temperature exceeds $10^{10} \mathrm{~K}$ in our models. We must caution that some of our results near the Schwarzschild radius are likely to be inaccurate, both because we use a Newtonian potential and because we do not include the expected sonic transition close to the hole. It would be of interest to include these effects self-consistently in future calculations.

\subsection{Massive Black Holes}


In Figure 4, we compare the critical accretion rates and the ion and electron temperatures for black holes of two very different masses, viz. $10 M_{\odot}$ and $10^{8} M_{\odot}$. For the same model parameters $(\alpha=0.3, \beta=0.5)$, the two cases show very similar behavior. This means that advection-dominated flows are nearly scale-free so that when the physical variables are scaled in terms of the relevant fiducial quantities (Eddington accretion rate, Schwarzschild radius) quantities like the critical accretion rate and the ion and electron temperatures are essentially independent of the mass. This is despite quite large differences in actual physical conditions like density, magnetic field strength, etc. The near invariance with respect to black hole mass suggests that some of the observable emission, such as bremsstrahlung radiation, from stellar mass black holes and supermassive black holes may be very similar, whenever these systems accrete in an advection-dominated mode. Not all features are similar, however. The synchrotron emission, for instance, is radiated at the critical frequency $\nu_{c}$ (equation 3.15), which is proportional to the magnetic field strength $B$ and therefore varies as $m^{-1 / 2}$ (see equation 2.15). This gives a fairly strong mass dependence. Note that the Shakura-Sunyaev thin disk solution has a characteristic blackbody temperature which scales as $m^{-1 / 4}$. This dependence is mid-way between those shown by the bremsstrahlung and synchrotron emission in the advection-dominated solution.

\subsection{Neutron Stars}

In Figure 5 we show our results for advection-dominated accretion on a neutron star. Apart from changing the mass of the accreting star to $1.4 M_{\odot}$ and the inner radius of the flow to $r_{i n}=r_{*}=2.5$ (corresponding to $R_{*}=10.5 \mathrm{~km}$ ), a crucial difference in this case is that we allow the star to have a hard surface. All the advected energy is assumed to be re-radiated at this surface. This radiation moves back through the hot accreting gas, and provides an additional source of photons for Compton cooling. In panels $a$ and $b$ of Fig. 5 we assume that the star radiates as a blackbody at temperature $T_{*}$ as given in equation (3.26), while in panel $c$ we consider for contrast a case where the radiation is emitted at essentially the electron temperature of the incoming gas, $T_{e}=10^{9} \mathrm{~K}$.

Black hole candidates among X-ray binaries in general have harder X-ray spectra than neutron star binaries. In recent times it has become accepted that the difference could be the result of extra cooling due to Comptonization of stellar photons in the neutron star systems (e.g. Sunyaev et al. 1991ab, Liang 1993, see also Sunyaev \& Titarchuk 1980). The results shown in Fig. 5 are an attempt to check this idea quantitatively, something that apparently has not been done so far.

Comparing Figs. 3 and 5 we see that there is indeed a substantial difference between the black hole and neutron star cases, especially in panels $a$ and $b$ where the stellar radiation is quite cool. We find that advection-domination is limited to much lower accretion rates in neutron stars compared to black holes. The difference arises primarily because of the very efficient Compton cooling by the stellar soft photons so that at a given relative accretion rate, a neutron star system cools more efficiently than a black hole system. It is therefore necessary to go to a lower $\dot{m}$ before the system can be advection-dominated. One consequence of the enhanced cooling is that, even though a black hole may be more massive (e.g. $10 M_{\odot}$ vs $\left.1.4 M_{\odot}\right)$, still for a given scaled accretion rate $\dot{m}$, neutron star and black hole systems may exhibit similar luminosities.

The other difference we notice between Figs. 3 and 5 is in the electron temperature. In general, $T_{e}$ is lower in the neutron star case than in the black hole case. Once again, cooling by soft photons is the reason. This difference may be expected to translate into fairly 
clear differences in the spectra, with accreting black holes likely to have harder spectra. This statement refers to the emission from the accretion flow. In addition, of course, the spectrum of an accreting neutron star will include unscattered soft thermal radiation from the stellar surface, whereas this component will be missing in the black hole case. One other consequence of the higher electron temperature in the black hole case is that it may lead to stronger pair effects at high $\dot{m}$, and this could produce significant differences between the two cases. However, pair effects are not important at accretion rates $\dot{m}<\dot{m}_{\text {crit }}$. We have calculated equilibrium pair densities in our solutions using the equations given in White \& Lightman (1989, see also Svensson 1984, Zdziarski 1985, Kusunose \& Takahara 1989), and in no case do we find a positron to ion ratio $n_{+} / n_{i}$ in excess of $\sim 10^{-3}$.

Panel $c$ in Fig. 5 corresponds to a case where there is the same amount of stellar radiation as in the previous two panels except that the radiation is at a temperature of $10^{9}$ $\mathrm{K}$ rather than the thermalization temperature of $\sim 10^{7} \mathrm{~K}$. We see that the critical lines move up a little and the electron temperature in the accretion flow is also a little higher. However, the results still do not quite match the corresponding results for a black hole. The point is that, even at such a high temperature, there is still significant Compton-cooling of the electrons by the stellar radiation. Therefore, we see that the effect of the stellar radiation is quite robust, and fairly insensitive to the temperature of the radiation.

Note that the critical $\dot{m}_{\text {crit }}$ lines in Fig. 5 have a peculiar jump at $r \sim 10^{5}$. At this radius, Comptonization of the stellar photons switches off suddenly (see below) and bremsstrahlung takes over as the dominant cooling.

\subsection{Dominant Cooling Mechanisms}

The ion temperature in our models is essentially determined by $f$, and for a large $f \rightarrow 1$, it is close to the virial temperature. The electron temperature, which is the temperature that is relevant for understanding the emission from the system, is however determined primarily by the cooling processes and the coupling between ions and electrons. We investigate in this section which cooling processes dominate as a function of radius in various accreting systems. We remind readers that our flows are assumed to have equipartition magnetic fields. These fields allow strong cooling through thermal synchrotron radiation under the right conditions. If such fields are absent $(\beta=1)$ or if they somehow do not participate in cooling, then the results will be quite different. Note in any case that we do not have nonthermal particles and therefore there is no nonthermal synchrotron emission in our model.

In Figure 6, we show as a function of $r$ the net cooling rates per unit volume due to bremsstrahlung $\left(q_{b r}^{-}+q_{b r, C}^{-}\right)$, synchrotron $\left(q_{\text {synch }}^{-}+q_{\text {synch,C }}^{-}\right)$and soft photon Comptonization $\left(q_{*, C}^{-}\right)$for various systems. In each case, we show the contributions due to these cooling processes along the critical line $\dot{m}_{c r i t}$ in the $\dot{m} r$ plane. The first two panels are for two accreting black hole models, one corresponding to $M=10 M_{\odot}$ and the other to $M=10^{8} M_{\odot}$. At large $r \gtrsim 10^{4}$, we see that the cooling is dominated by bremsstrahlung. Recall that this is approximately the region where the critical accretion rate $\dot{m}_{\text {crit }}$ can be calculated using the simple relation given in eq (4.1). At lower radii, the electron temperature becomes large, $T_{e} \gtrsim 10^{9} \mathrm{~K}$, and the quasi-relativistic thermal electrons discover new channels of cooling through synchrotron emission and Comptonization. Therefore, for $r \lesssim 10^{2}$, synchrotron emission dominates over bremsstrahlung. This switchover is partially responsible for the deviation of the critical $\dot{m}_{c r i t}$ line from eq (4.1) (see Fig. 1). Note that there is very little difference between the $10 M_{\odot}$ case and the $10^{8} M_{\odot}$ case, confirming the scale-free nature of the black hole solutions. 
Figure 7 shows a more detailed representation of the various cooling processes for an accreting $10 M_{\odot}$ black hole. For this figure we scanned the entire $\dot{m} r$ plane below the critical line, and at each point we determined which of the four cooling terms, $q_{b r}^{-}, q_{s y n c h}^{-}, q_{b r, C}^{-}$, $q_{\text {synch }, C}^{-}$, dominates. We have labeled the zones where these terms dominate as $1,2,3$, 4 respectively in the figure. There are two clear trends. First, synchrotron-related cooling dominates at lower radii and bremsstrahlung-related cooling at larger radii as we have already noted. Second, we see that the importance of Comptonization increases at higher values of $\dot{m}$. The cooling due to Comptonization depends on the Compton $y$ parameter which varies sensitively with the electron scattering optical depth $\tau_{e s}$ through the factor $P$ given in Eq (3.20). The optical depth is essentially proportional to $\dot{m}$ (see eq 2.15) and therefore as $\dot{m}$ increases there is a catastrophic increase in Compton cooling. Indeed, at all radii $\lesssim 10^{3}$, the position of the critical $\dot{m}_{c r i t}$ is determined almost entirely just by the requirement that the optical depth be of order unity (which at these electron temperatures is equivalent to the Compton $y$ parameter being of order unity).

Figure 7 gives a convenient method of gaining a qualitative understanding of the cooling processes and spectrum of an advection-dominated accretion flow. A system with a specific $\dot{m}$ corresponds to a horizontal track in this figure. By identifying which zones are present along the track, and at what radii, it is possible to obtain a quick idea of the radiation emission from the system. Analogous results are presented by Rees et al. (1982) for twotemperature plasmas in their ion torus models. They present their results as a function of $T_{e}$ and $r$ for fixed $\dot{m}$. In our model, $T_{e}$ is not a free parameter but is determined uniquely by the various processes that are modeled.

Panel c in Fig. 6 shows the results for an accreting neutron star. We see that, in this case, the cooling is dominated by the Comptonization of soft photons over a wide range of radius all the way out to $r \sim 10^{5}$. In these models synchrotron cooling is not competitive even at the smallest radii. This is because of the lower electron temperature compared to the black hole models. At $r \sim 10^{5}$, the electron temperature is equal to the temperature of the stellar emission and beyond this radius the soft photon cooling shuts off and bremsstrahlung cooling takes over. Actually, at these radii there will be Compton heating of electrons which we have not included in our models. This could be an important effect (Ostriker et al. 1976, see also Grindlay 1978 who discusses the effect of Compton-heating on the thermal pre-heating in X-ray burst sources), and we hope to include it in future work.

\subsection{Stability of the Advection-Dominated Branch}

Two features of our advection-dominated solutions are that they are optically thin, and that (at large radii at least) they are dominated by bremsstrahlung cooling. Accretion flows are normally thermally unstable precisely under these conditions (Pringle et al. 1973, Piran 1978). The reason for the instability is that bremsstrahlung cooling is proportional to the square of the density. If the flow is perturbed to a slightly hotter state than the equilibrium, the density decreases and this leads to less efficient cooling. The reduced cooling then causes the disk to become yet hotter and there is a runaway instability.

The important new feature of our models is that we have included advection in the equations, whereas the thermal stability analyses referred to above did not include this effect. Abramowicz et al. (1988) (see also Honma et al. 1991) showed that advection causes high $\dot{m}$ optically thick flows to become stable even though radiation pressure dominates in these systems and one would normally expect the Lightman-Eardley (1974) instability 
to operate. More recently, models of boundary layers calculated by Narayan \& Popham (1993) had indicated that the thermal instability has a close connection to advection even in optically thin flows. In models of CVs, these authors noticed that the boundary layer becomes optically thin at low accretion rates, and goes thermally unstable. As a consequence of the instability, the gas in the models heats up rapidly to very high temperatures, but despite this, Narayan \& Popham (1993) were able to obtain steady state solutions. A feature of their solutions is that, once the temperature of the unstable gas approaches the virial limit, the flow switches to an advection-dominated mode of accretion and a perfectly reasonable steady state equilibrium flow results. The implication is that, even though the accreting gas is thermally unstable, it is nevertheless able to survive through advection. We explore the issue in some detail here. Because this is an extremely important and central result of this paper, we discuss the thermal stability of the various solutions in considerable detail. An analogous but briefer discussion can be found in Abramowicz et al. (1995).

Figure 8a shows some results corresponding to a specific case with $m=10, \dot{m}=10^{-3}$, $r=10^{3}, \alpha=0.3, \beta=0.5$. In these calculations we allowed $f$ to vary over a wide range of values from $f=10^{-6}$ all the way up to $f \rightarrow 1$. For each value of $f$, we ignored the energy balance of the ions as described by equation (3.34), but we solved all the other equations. In particular, we made sure that eq (3.35) is satisfied so that the thermal balance of the electrons is maintained, i.e. the rate at which the electrons are heated by Coulomb transfer of energy from the ions is exactly compensated by the rate of cooling of the electrons. Having done this, we then checked the thermal balance of the ions by looking at the terms in eq (3.34). Recall that the parameter $f$ is defined such that the advected energy is given by $q^{a d v}=f q^{+}$. In thermal balance, the cooling rate of the ions, which is given by $q^{i e}=q^{-}$, should be equal to $q^{+}-q^{a d v}=(1-f) q^{+}$; in other words, the ratio $q^{-} /(1-f) q^{+}$should be equal to unity. Any deviation of this ratio from unity indicates that the ions are not in thermal balance. The sense of the deviation, i.e. whether the ratio is greater than or less than unity, tells us whether the ions will respond by cooling or heating.

In Fig. 8a we show the calculated values of the ion temperature $T_{i}$ as a function of the ratio $q^{-} /(1-f) q^{+}$. We obtain a characteristic S-shaped curve which intersects the equilibrium condition, $q^{-} /(1-f) q^{+}=1$, at three points marked A, B, C. These three points represent three equilibrium solutions which satisfy all the equations, including eq (3.34). All three solutions are valid solutions of the equations, but they have different stability properties.

Consider the solution A which has the lowest value of $T_{i}$. This is the standard thin accretion disk solution. At the position of this solution, we see that the line describing $T_{i}$ vs $q^{-} /(1-f) q^{+}$increases upwards to the right. Let us imagine taking this equilibrium and perturbing it to a slightly hotter ion temperature $T_{i}$, and let us assume that the electron temperature quickly adjusts so as to maintain eq (3.35). The system will then move to a new position on the curve corresponding to the new $T_{i}$. Because of the positive slope of the line, we see that the system will go to a state with $q^{-} /(1-f) q^{+}>1$, i.e. with $q^{i e}+q^{a d v}>q^{+}$. This means that the right-hand side of eq (3.34) will be larger than the left hand side, and the cooling of the ions will dominate over the heating. Obviously, the gas will respond by cooling and this means that $T_{i}$ will automatically decrease. Similarly, if we perturb the system to a lower value of $T_{i}$ from the equilibrium, then $q^{-} /(1-f) q^{+}$ will decrease below unity and the gas will heat up and go back towards the equilibrium solution. Thus, the thin disk solution A is thermally stable.

Consider next the solution marked B in Fig. 8a. Here the curve increases upward to the left. Therefore, by the same argument as above, this solution is unstable. For instance, 
if we set up an equilibrium flow corresponding to this solution and perturb it to a slightly hotter state, then the ion cooling will reduce relative to the heating. The gas will therefore heat up further, and we will have a classic thermal instability (Pringle et al. 1973, Piran 1978). The solution B is the standard hot solution (SLE) which has been known for many years and which has been recognized to be thermally unstable.

Finally, consider the upper most solution marked $\mathrm{C}$ with the highest value of $T_{i}$. This is our new advection-dominated branch. At the position of this solution, we find that the curve once again rises upwards to the right, as in solution $\mathrm{A}$. By the same arguments as before this solution is therefore stable. The interesting thing is that this flow is even more optically thin and more bremsstrahlung dominated than solution B, and one would normally expect this solution to be violently unstable. The secret to its stability is the presence of the advection term in our equations.

To prove this assertion, we show in panel $\mathrm{b}$ a calculation where for the same system we calculate the ratio $q^{-} / q^{+}$. The point is that if there is no advection, then we require $q^{-}=q^{+}$, and so the ratio $q^{-} / q^{+}$has to equal unity in equilibrium. For easy comparison with previous discussions of the thermal instability in the literature we include only electron-ion bremsstrahlung cooling in the calculation. We see that the curve in Fig. 8b satisfies the equilibrium condition $q^{-} / q^{+}=1$ at only two points, identified as $\mathrm{A}$ and $\mathrm{B}$. Solution $\mathrm{A}$ is stable and corresponds to the standard thin accretion disk as before. Solution B is unstable, again as before. If such a system were set up in a state where the ion temperature is hotter than the equilibrium temperature of solution B, then it would heat up without limit and would never find a thermally stable state. This is clearly a paradoxical situation. In contrast, if a system is described by Fig. 8a, then, regardless of what initial state it begins with, the flow can always find a stable solution. This indicates the great importance of including advection when one is discussing the thermal stability of accretion disks.

The above discussion was for a system with a fixed $\dot{m}$. In Fig. 2 we showed how the three branches behave as $\dot{m}$ is varied. There we plotted $f$ versus $\dot{m}$ for each of the branches. Figure $8 \mathrm{c}$ shows the same branches again, this time showing how the ion temperature $T_{i}$ varies along the three solutions. The three branches are labeled A, B, C as in the panel a. Finally, in Fig. 8d we show the same information as in Figs. 2, 8c in yet another representation where we plot $\dot{m}$ vs the surface density $\Sigma=\rho H$ of the flow. This kind of representation is commonly used in the literature in discussions of stability. We have indicated the two stable branches by solid lines and the unstable branch by a dotted line. An analogous figure can be found in Abramowicz et al. (1995) and Chen (1995). Note that at very low $\dot{m} \lesssim 10^{-6}$, we find $T_{i} \lesssim 10^{4} \mathrm{~K}$ in the $\mathrm{A}$ branch. At these low temperatures, the cooling is more complicated than our simple model because of $\mathrm{H}$ recombination, and the effect of molecules, dust, etc. We have not tried to include these opacities in our model because we are at this point interested merely in demonstrating the basic physics which distinguishes the various branches.

Finally we would like to emphasize that the systems we are modeling here do not undergo a thermal limit cycle. In discussions of the thermal instability associated with hydrogen ionization (Smak 1984, Mineshige \& Wheeler 1989) an S curve in the $\dot{m}$ vs $\Sigma$ plane is often shown. Abramowicz et al. (1995) show an analogous S curve corresponding to their high $\dot{m}$ optically thick disks around black holes. In both those cases, the topology of the $\mathrm{S}$ is different from that in Fig. 8d. As a result, for certain choices of $\dot{m}$, the only solution available in those problems is an unstable one. It can be shown that the accretion will then be forced into a limit cycle behavior where the flow oscillates between the two stable branches. In our case, there is no such problem. Over the ranges of $\dot{m}$ and $R$ we 
consider, there is always at least one stable solution available and therefore there is no need for a limit cycle. In fact, for a wide range of $\dot{m}$ we actually have two stable solutions, which brings up an important question: Which of the two states does the system choose? We discuss this issue in the next section.

We conclude this section with two comments. First, we note that the thin accretion disk solution becomes unstable at sufficiently high $\dot{m}$ because of the effect of radiation pressure (Lightman \& Eardley 1974). Our analysis cannot demonstrate this instability because we have neglected radiation pressure (for simplicity). The analysis of Abramowicz et al. (1988) does include radiation pressure and they see the effects of this instability as an $\mathrm{S}$ curve in the A branch near the top right corner of Fig. 8d.

Our second comment concerns the viscous stability of the solutions. In addition to the thermal instability discussed above in detail, accretion disks are also prone to a secular viscous instability whenever the slope $d \dot{m} / d \Sigma$ is negative (e.g. Lightman \& Eardley 1974, Piran 1978). Figure 8d shows that all three branches of solutions we have considered have $d \dot{m} / d \Sigma>0$ and are therefore viscously stable, except in very small regions around the turning points. The viscous stability of the A and B branches was already known (e.g. Wandel \& Liang 1991). We see that our new advection-dominated branch is also viscously stable. 


\section{When Does Optically Thin Advection-Dominated Accretion Occur?}

In $\S 4$ we calculated the critical $\dot{m}_{c r i t}$ for optically thin advection-dominated accretion and presented results for accreting black holes and neutron stars (Figs. 3-5). All points on the $\dot{m} r$ plane which lie below the calculated $\dot{m}_{c r i t}(r)$ line have a stable advection-dominated branch of solutions. However, over the entire region between the upper critical line $\dot{m}_{\text {crit }}$ and the lower line $\dot{m}_{c r i t}^{\prime}$ (which is at an extremely low accretion rate, see eq 4.2), a second solution is also allowed, namely the standard cooling-dominated thin disk solution. Which of the two solutions will an accretion flow actually choose? In the following three subsections we suggest three answers to this question, the first of which is the most conservative and the last the most radical and sweeping. The three suggestions are not mutually exclusive.

\subsection{Advection-Domination Triggered by the Lightman-Eardley Instability}

Our first argument is based on the well-known fact that the thin disk solution is unstable at high $\dot{m}$ and small $r$ due to the effect of radiation pressure (Lightman \& Eardley 1974). Whenever radiation pressure dominates over gas pressure, a thin accretion disk becomes both thermally and viscously unstable. The transition to radiation pressure domination happens across the following line in the $\dot{m} r$ plane (Frank et al. 1992),

$$
\dot{m} \approx 4.3 \times 10^{-3} \alpha^{-1 / 8} m^{-1 / 8} r^{21 / 16},
$$

which is shown in Fig. 9a,b for an accreting $10 M_{\odot}$ and $10^{8} M_{\odot}$ black hole. The critical advection-dominated line $\dot{m}_{\text {crit }}$ is also shown in the figure. We see that there is a small triangular-shaped area towards the left of the figure where a thin disk suffers the LightmanEardley instability but where a stable advection-dominated solution is possible. We argue that, in this region of parameter space, accretion has to occur in the advection-dominated mode since this is the only stable solution available. Thus, our most conservative proposal is that the optically thin advection-dominated solutions discussed in this paper are restricted to the narrow triangular regions shown in Fig. 9 (plus the region $\dot{m}<\dot{m}_{c r i t}^{\prime}$, which is at much too low an accretion rate to be of practical importance). A proposal similar to this is widely discussed in the literature, where it is proposed (e.g. SLE) that a disk which is unstable by the Lightman-Eardley mechanism will switch to the SLE hot solution. The main difference in what we propose is that the relevant hot state is not the SLE solution but rather our new advection-dominated solution. Incidentally, note that our advection-dominated solutions are always gas pressure dominated and therefore are in no danger from the Lightman-Eardley instability. This is why it is appropriate for us to neglect radiation pressure in our models of the advection-dominated state.

As an aside we note that the Lightman-Eardley (1974) instability operates only if the viscosity coefficient is taken to be proportional to the total pressure (gas plus radiation pressure). The instability is absent if viscosity is proportional only to the gas pressure. Although such a prescription has been motivated by some earlier work (e.g. Lightman \& Eardley 1974, Coroniti 1981, Stella \& Rosner 1984), it is not clear how realistic such a prescription is, especially in view of the work of Balbus \& Hawley (1991) on magnetic viscosity.

\subsection{Effect of Initial Conditions}


As a slightly less conservative proposal, we suggest that an advection-dominated flow occurs whenever the initial conditions of the accreting material at the outer radius correspond to an advective form. Consider as a specific example the case shown in Fig. 10a which consists of a $10 M_{\odot}$ black hole accreting at a mass accretion rate of $\dot{m}=10^{-2}$. The track of the system, shown by a dotted line in the figure, intersects the critical line at $r=r_{c r i t}=10^{5.5}$ (the filled circle). Suppose the black hole accretes from a companion star and suppose the outer radius where the material is injected lies at $r_{\text {out }}<r_{\text {crit }}$. If the material comes in very hot, i.e. close to virial, or if the material comes in cold but undergoes an adiabatic shock when it meets the accretion disk and heats up to the virial temperature, then the initial conditions will place the gas in the advection-dominated branch. Since this is a stable solution, we propose that the flow will continue inwards along the same branch. In other words, even though a thin cooling-dominated disk is allowed the gas does not discover this branch of solutions because of its initial conditions. If, however, the accretion begins with $r_{\text {out }}>r_{\text {crit }}$, then regardless of whether the initial conditions are hot or cold, the incoming gas will initially have to form a thin disk. This is because $\dot{m}>\dot{m}_{\text {crit }}\left(r_{\text {out }}\right)$ and no advectiondominated solution is allowed. Once the flow is trapped in the thin disk branch at $r=r_{\text {out }}$ it will follow this branch all the way down to a small radius until either it hits the horizon or passes into the Lightman-Eardley unstable zone. Thus, in this proposal, everything depends on initial conditions.

\subsection{Advection-Dominated State Preferred Whenever it is Available}

We come now to our most radical suggestion, which we feel is quite possibly the way nature really works. We speculate that over much of the region below the critical line, i.e. whenever $\dot{m}<\dot{m}_{c r i t}(r)$, an accretion flow selects the advection-dominated branch. We base this speculation on a feature of the vertical structure of thin disks which makes coolingdominated solutions prone to a surface instability.

A number of researchers have attempted to go beyond the "one-zone" solutions that are common in thin accretion disk theory in order to find detailed solutions for the vertical structure of these disks (Shaviv \& Wehrse 1986, 1989, Adam et al. 1986). The most complete work so far is by Shaviv \& Wehrse (1989) who set up a self-consistent set of equations for hydrostatic equilibrium, energy generation, and radiative transfer, and numerically calculated the vertical solution. One of the surprising results of their work is that for many reasonable forms of the energy generation law, they find that the uppermost layers of the disk become thermally unstable so that it is impossible to find steady state solutions. We note, however, that the thermal instability does depend on the details of the viscous energy generation and the opacity in the optically thin surface layer. The explanation of the instability is straightforward. In any solution for the vertical structure, the outermost layer (the atmosphere) has very low density and therefore has poor cooling efficiency. This region is also optically thin to the outside. If viscosity dissipates any energy in this low density optically thin material, the gas has no way of radiating the energy and therefore has to heat up. This is exactly the thermal instability discussed earlier except that it is restricted to the outermost layer of the disk at optical depth $\tau \lesssim 1$. Any irradiation of the surface will only enhance the instability.

What happens to the unstable surface gas? We suggest that it will keep on expanding until it becomes almost virial. Once virial, the gas will be forced to switch to an advectiondominated mode and will form an advective differentially-rotating accreting corona on top of the disk. Moreover, the optical depth of the material will decrease drastically because its 
radial velocity will be much greater than the velocity in the disk. Once the top layer of the disk has boiled off, what about the remaining gas in the disk? A new layer on the surface of the disk, down to optical depth unity, will next become unstable and it will take off into the corona. In this manner the disk surface will continue to boil away until the entire disk has been converted into an accreting corona. The final state is of course what we call our advection-dominated branch of solutions. Note that the instability which we invoke is explicitly a property of the vertical stratification of the disk and is beyond the scope of the height-integrated equations we have used in this paper. This is why our analysis of $\S 4.7$ showed the thin disk branch to be stable. Incidentally, since the advection-dominated flow is much more homogeneous as a function of height (see the solutions in Paper 2) and since it is anyway insensitive to cooling, it is not expected to be unstable by this mechanism.

If we accept the above instability, then we believe that any thin disk which is prone to a vigorous surface instability and which has $\dot{m}<\dot{m}_{\text {crit }}$ will over a period of time evaporate into a corona. The critical question is: Under what conditions does the surface instability act with sufficient speed to ensure complete evaporation of the thin disk? This is a difficult question, whose answer depends on the vertical stratification of the viscous stress and on the details of the optically thin cooling in the surface layer. The topic is beyond the scope of this paper, but we believe there is a real possibility that, over a wide region below the critical $\dot{m}$ lines which we have calculated in this paper, the true equilibrium flow is the advection-dominated solution and that no thin disk flows should be seen.

What does the surface instability do to accretion flows which lie above the critical line? In these cases again the surface layers of the disk will become unstable and will evaporate to form a corona. However, the entire disk cannot evaporate because there is no advectiondominated solution which can handle such a large $\dot{m}$. We suggest therefore that the disk will evaporate only until the corona has the maximum $\dot{m}$ allowed at the given $r$. The rest of the mass will remain in the cool disk. We thus envisage a sandwich structure where the corona will be maximally advection-dominated, while the central thin disk will be coolingdominated. We obtained solutions similar to this in Paper 2 where some of our self-similar flows had a high density equatorial flow coexisting with a very hot low density advective corona on top. The role of pairs in such a structure could be important but is uncertain at this point.

Figure 10b shows schematically how the proposal works for the specific example considered earlier. Let us suppose that $\dot{m}=10^{-2}$ and $r_{\text {out }}>r_{\text {crit }}$. Let us also assume that evaporation occurs very rapidly at all radii. At radii $r>r_{c r i t}$, the accretion rate lies above the critical $\dot{m}_{c r i t}(r)$. Therefore, in this region, we suggest that we will have a saturated corona with an accretion rate given by $\dot{m}_{c o r}=\dot{m}_{c r i t}$, and that the excess material will flow through the equatorial thin disk with $\dot{m}_{d i s k}=\dot{m}-\dot{m}_{c r i t}$. As $r$ decreases, the relative fraction of the mass in the corona increases and that in the disk decreases. When $r$ reaches the critical value $r=r_{\text {crit }}$, all the mass in the disk will have evaporated into the corona. Below $r_{c r i t}$, the accretion occurs purely in an advection-dominated mode. Thus, the flow has three zones: there is a thin disk zone at large $r>r_{\text {crit }}$, a corona above the thin disk over the same range of $r$, and a fully advection-dominated zone at small $r<r_{\text {crit }}$.

Meyer \& Meyer-Hofmeister (1994) proposed a new model of low $\dot{m}$ CVs which is very similar to the one we describe. Their systems have normal thin disks at large radii while at smaller radii they assume that the flow is entirely in a hot nearly virial state, i.e. in an advection-dominated state. By postulating a hole in the accretion disk at small radii they show that they can explain naturally some puzzling features in CV observations such as the so-called UV delay. The identification of a stable advection-dominated solution branch in 
this paper provides strong support to the Meyer \& Meyer-Hofmeister proposal. At the same time, we view their success in explaining CV observations as support for our suggestion that the advection-dominated flow is the most natural and stable form of accretion possible and that accretion flows will often choose this form when it is available.

Of course, this is just a qualitative proposal at the present time and will need to be compared with detailed calculations. For instance, the maximum $\dot{m}_{c r i t}$ which the corona can handle will not be exactly the $\dot{m}_{\text {crit }}$ which we have calculated in this paper. This is because the equatorial thin disk is a source of soft photons which can cause additional Compton cooling (e.g. SLE). Also, we need to show that the time scale on which a thin disk evaporates into the corona is shorter than the accretion time. Only then is the surface instability of any importance. A quantitative discussion of these issue is beyond the scope of this paper. 


\section{Summary and Discussion}

Advection-dominated flows occur when the local cooling time scale becomes longer than the accretion time scale, so that most of the dissipatively liberated energy is advected inward with the accreting gas rather than being radiated. The result is that the gas temperature becomes almost virial and the luminosity of the system falls well below the standard $G M \dot{M} / R_{*}$ that is normally expected from accretion. As we have discussed in Papers 1 and 2, advection-dominated flows can in principle occur either in systems with very high mass accretion rates (Begelman 1978), where the photon diffusion time scale is very long, or in systems with very low mass accretion rates (Rees et al. 1982), where the local optically thin cooling time scale becomes very long. In this paper we concentrate on the latter case.

Our model of the accretion flow combines the dynamical equations of Papers 1 and 2 (summarized in §2) with a comprehensive description of cooling processes which includes bremsstrahlung, synchrotron, and Comptonization (§3). We model the gas as a twotemperature plasma (following SLE) where the ions and electrons are allowed to have different temperatures, determined by individual thermal balance equations for each species. We consider accretion onto black holes and neutron stars, the distinction being that in one case all the advected energy disappears through the horizon whereas in the other the energy is thermalized and reradiated at the stellar surface. In our models, we assume that the flows contain roughly equipartition strength magnetic fields whose strength is estimated through a parameter $\beta$ (see eqs 2.6, 2.10). Hot electrons can cool through synchrotron emission in the magnetic field. The field may also be the agency whereby angular momentum is transported, but we do not model this process in detail. Instead we use a standard $\alpha$ prescription for viscosity, where the parameter $\alpha$ is assumed to include the effects of hydrodynamic turbulence/convection and magnetic stresses. Occasionally, in the literature, radiative viscosity is discussed in the context of accreting black holes and neutron stars (e.g. Loeb \& Laor 1992, Miller \& Lamb 1993). As we show in Appendix B, this viscosity is not important for the flows considered in this paper.

The main results of our calculations are described in $\S 4$. By numerically solving the equations we find that the model gives three distinct branches of equilibria $(\S \S 4.2,4.7)$, two of which are stable and one of which is thermally unstable. One of the stable branches is the standard cooling-dominated thin accretion disk solution which is much discussed in the literature (Shakura \& Sunyaev 1973, Novikov \& Thorne 1973, Lynden-Bell \& Pringle 1974). The unstable branch corresponds to a hot optically thin solution which is again much discussed in the literature (SLE, Luo \& Liang 1994, and references therein). The third branch, which corresponds to a hot, optically thin, advection-dominated flow, is a new solution which comes out of our analysis and which is the principal focus of this paper. This solution is thermally stable. Moreover, as we demonstrate in $\S 4.7$, it is the introduction of advection which allows the solution to be stable despite being optically thin. This branch has also been discussed by Abramowicz et al. (1995) and Chen (1995) who consider a simplified version of the theory presented here (see $\S 4.1$ ).

Is our new solution branch really new? Certainly, to the extent that we have for the first time included advection and treated the dynamics of the flow consistently, the advectiondominated solution described in this paper and in Abramowicz et al. (1995) is new. But, even more fundamentally, it is our impression that the existence of two hot solutions (only one of which is thermally stable) was not appreciated until now. Hot solutions have been quite popular for many years, beginning with the important work of SLE, but these solutions are calculated under a local cooling assumption, $q^{-}=q^{+}$. As a consequence, the calculations 
invariably produce the thermally unstable solution branch mentioned above. The ion torus model of Rees et al. (1982) comes closer to our new advection-dominated solution since the model specifically avoids the condition $q^{-}=q^{+}$. However, their paper does not carry out a complete analysis of the dynamics and it is not clear if the proposed solution is in fact our advection-dominated solution. Also, Rees et al. do not explain the relationship between their solution and the previously known hot SLE solutions. Indeed, in much of the accretion literature, the ion torus is considered to be a variant of the SLE hot solution (e.g. Frank et al. 1992, p233) rather than as an independent solution branch.

Using our model, we have explored in some detail the properties of the optically thin advection-dominated branch of solutions. For each choice of the stellar mass $M$, the viscosity parameter $\alpha$, and the equipartition parameter $\beta$ (eq 2.6), we have determined a critical line $\dot{m}_{c r i t}(r)$ in the $\dot{m} r$ plane. Here, $\dot{m} \equiv \dot{M} / \dot{M}_{E d d}$ is the accretion rate scaled to the Eddington rate with an efficiency $\eta_{\text {eff }}=0.1$ (see eq 2.13) and $r=R / R_{S c h w}$ is the radius scaled to the Schwarzschild radius (eq 2.14). The critical line $\dot{m}_{c r i t}(r)$ gives at each $r$ the maximum accretion rate above which the optically thin advection-dominated branch of solutions terminates. Figures 3-5 show our results for accreting black holes of mass $10 M_{\odot}$ and $10^{8} M_{\odot}$ and for neutron stars. The critical lines are to be interpreted as follows. Any given system with a specified accretion rate $\dot{m}$ corresponds to a horizontal line on the diagram. If the system has a high accretion rate so that $\dot{m}$ lies entirely above the critical line then it cannot be advection-dominated. However, if part of the flow lies below the critical line, then the flow is allowed to be advection-dominated over that range of $r$.

In addition to the critical line $\dot{m}_{\text {crit }}$ discussed above and shown in Figures $3-5$, we show that there is a second limit, which we designate $\dot{m}_{c r i t}^{\prime}$ and which is located at an extremely low accretion rate, $\dot{m}_{c r i t}^{\prime} \lesssim 10^{-10}$ (see eq 4.2 ). The two critical lines divide the $\dot{m} r$ plane into three distinct zones. Above $\dot{m}_{c r i t}$, there is no advection-dominated solution. Below $\dot{m}_{\text {crit }}^{\prime}$, the thin disk solution is unstable, and the advection-dominated flow is the only solution allowed. Between the two critical lines, however, and this covers a very wide range of astrophysically interesting parameters, both the advection-dominated solution and the standard cooling-dominated thin disk solution are apparently allowed. Which solution does nature actually pick?

We discuss this issue in $\S 5$ and argue that whenever the thin disk solution is unstable for any reason the gas will automatically go into the advection-dominated mode. In certain regions of the $\dot{m} r$ plane (see eq 5.1) a cooling-dominated thin disk becomes radiation pressure dominated and in consequence is unstable if the viscosity is proportional to the total pressure (Lightman \& Eardley 1974). In these regions we believe that the flow will automatically switch to the advection-dominated solution. A similar idea has been widely discussed in the literature (e.g. SLE), except that in most previous discussions a thin disk which is unstable to the Lightman-Eardley instability is assumed to switch to an SLE-type hot solution, which is itself unstable. The new feature here is that we suggest that the final state is our advection-dominated hot state.

In addition to the well-known Lightman-Eardley instability, we argue that, even in other regions of the $\dot{m} r$ plane, a thin disk has a different kind of thermal instability which operates on its surface layers and may cause the disk to evaporate in the vertical direction. We therefore speculate that thin disks are probably unstable over much of the region below the critical line $\dot{m}_{c r i t}(r)$ and that many flows below this line are advection-dominated. Further, we argue that even systems with $\dot{m}>\dot{m}_{\text {crit }}$ may have some part of the mass accretion occurring in an advection-dominated corona. These ideas are summarized in Fig. 
10, and are similar to suggestions made recently by Meyer \& Meyer-Hofmeister (1994) in the context of accretion disks in CVs.

Thermally unstable accretion disks with multiple equilibria have been discussed in other contexts (Smak 1984, Abramowicz et al. 1988, Mineshige \& Wheeler 1989), and in most cases it is argued that they undergo a thermal limit cycle. The limit cycle is used to explain the observed variability of the systems. Although we have multiple equilibria, the flows described in this paper do not have any tendency to set up a limit cycle, as we discuss in $\S 4.7$. Note, however, that at the higher accretion rates considered by Abramowicz et al. (1988), limit cycles are indeed a possibility, but their discussion refers to different solution branches than those considered here.

Generally we find that advection-dominated flows occur for mass accretion rates $\dot{m} \lesssim 10^{-1}$ $10^{-3}$ for the systems we have considered. The results however depend on the value of $\alpha$ and to a lesser extent $\beta$. There is also a large difference depending on whether the accreting star is a black hole or a neutron star (see Figs. 3-5), as anticipated in some previous papers (e.g. Sunyaev \& Titarchuk 1980, Sunyaev et al. 1991ab, Liang 1993). At small $r$, we obtain a critical accretion rate of $\dot{m}_{c r i t} \sim \alpha^{2}$ for black hole systems, and $\dot{m}_{c r i t} \sim 0.1 \alpha^{2}$ for neutron star systems.

All of our advection-dominated solutions have certain common features. The ion temperature is always close to virial at all radii. Since the flow is advection-dominated, most of the energy which is released by viscosity is retained in the gas, and the ions therefore have to heat up to close to the virial temperature. Our solutions are thus similar to the ion torus model of Rees et al. (1982), except that our flows are more nearly spherical than toroidal (see Paper 2). At large radii, the electrons are in near thermal equilibrium with the ions and are therefore virial. However, at radii $r \lesssim 10^{3}$ when the ion temperature exceeds $10^{9} \mathrm{~K}$, the electron temperature deviates from the ion temperature. This is because, above $10^{9} \mathrm{~K}$, the ion-electron coupling via Coulomb collisions becomes weak and at the same time the electrons are able to cool by a variety of processes including synchrotron emission and Compton cooling. The efficient cooling keeps the electrons at a roughly constant temperature independent of $r$, whereas the ions decouple from the electrons and continue to become hotter as $r$ decreases.

The two-temperature feature of our solutions is largely a consequence of our assumption that the only coupling between ions and electrons is via Coulomb scattering. If there are non-thermal modes of energy transfer from ions to electrons (Phinney 1981), for example by the collective mechanism discussed by Begelman \& Chiueh (1988, hereafter BC), then the results could be strongly modified. We discuss this important issue in Appendix A. The $\mathrm{BC}$ mechanism makes use of wave interactions in regions of large density perturbations to transfer energy directly from waves to electrons. We consider two cases in Appendix A. If the density perturbations are caused by a normal Kolmogorov-like turbulent cascade, then we show that for most cases of interest, the heating of electrons via Coulomb collisions is stronger than that due to the $\mathrm{BC}$ mechanism. When either $\alpha$ or mass accretion rate is very small, heating by the BC mechanism may dominate over the Coulomb heating. Even in this case, however, the viscous heating rate of ions is much larger than the BC heating rate of electrons except for very small values of $\alpha$, which suggests that the twotemperature assumption is justified nearly in all cases. Therefore, it is appropriate to neglect non-thermal heating of electrons. In the alternate case, where most of the dissipation occurs in collisionless shocks, we still find that the BC mechanism is unimportant so long as the accretion star is a stellar-mass black hole. However, if the star is a supermassive black hole, then it appears that the $\mathrm{BC}$ non-thermal heating of electrons wins over Coulomb heating. 
Therefore, depending on the nature of the dissipation, our results may or may not be valid for AGN.

One of our interesting results is that black hole models are almost scale-independent. For the whole range of masses from $M=10 M_{\odot}$ to $10^{8} M_{\odot}$ we find that the position of the critical line $\dot{m}_{c r i t}(r)$ as well as the dependences of the ion and electron temperatures on scaled radius $r$ are nearly the same. Individual physical quantities (volume density, magnetic field strength, etc.) do vary by large factors from stellar mass to supermassive black holes but the electron temperatures remain virtually identical. This scale-free behavior is true only for the advection-dominated branch of solutions. Cooling-dominated thin disks are known to depend on the mass of the central star, e.g. the surface temperature varies as $M^{-1 / 4}$. The scale-free nature of advective flows may explain some of the similarities that are observed between galactic black hole X-ray binaries and active galactic nuclei. The bremsstrahlung emission at least in the two cases must have similar characteristics. The synchrotron radiation however will be at very different frequencies, since the self-absorption frequency $\nu_{c}$ (equation 3.15) scales as $M^{-1 / 2}$.

Our calculations indicate that there are strong differences between advection-dominated accretion flows around black holes and those around neutron stars (compare Figs. 3 and 5). The difference arises primarily because in the latter case all the advected energy is reradiated as blackbody radiation from the stellar surface, whereas in the former the energy is lost into the hole. This point has been emphasized before (e.g. Sunyaev et al. 1991ab, Liang 1993) and leads to several effects.

(i) The spectrum of an accreting neutron star will have an additional cool component due to the radiation from the star, which will be missing in an accreting black hole.

(ii) Cooling is more effective in the neutron star case because the soft photons from the star Compton-cool the electrons in the accreting gas very effectively. We see clear evidence for this in our calculations, thereby confirming previous suggestions of the effect (Sunyaev et al. 1991ab, Liang 1993).

(iii) As a result of (ii), the critical line for an accreting neutron star lies at a much lower value of $\dot{m}$ than for a black hole. Because of this shift, we suggest that a significantly larger fraction of black hole binaries in the Galaxy may be in an advection-dominated mode compared to neutron star binaries.

(iv) One of the features of the advection-dominated branch is that the radiation luminosity is low (Phinney 1981, Rees et al. 1982). Combining this with the previous points, we suggest that the majority of black hole binaries may be unusually dim for their accretion rate. This may have some implications for understanding transient X-ray sources, many of which are believed to be accreting black holes. It may also explain why black hole X-ray binaries typically have similar luminosities as neutron star binaries even though the central stars in the former are believed to be much more massive. Figure 11 illustrates quantitatively the difference between accreting black holes and neutron stars. The two panels show the luminosities of the two systems as functions of $\dot{m}$. We see that the luminosity of the black hole accretor drops rapidly with decreasing $\dot{M}$, giving extremely low radiative efficiency for $\dot{m} \ll \alpha^{2}$. Roughly, the luminosity scales as $L \sim 0.1 \dot{M} c^{2}\left(\dot{m} / \alpha^{2}\right)$. In contrast, an accreting neutron star has a more or less constant efficiency of $\sim 20 \%$, independent of $\dot{m}$, i.e. $L \sim$ $0.2 \dot{M} c^{2}$. Of course, at low $\dot{m}$, the accretion flow is highly advection-dominated in the neutron star case as well, as shown by the dotted line in Fig. 11. However, all the advected energy is re-radiated from the surface of the star, and therefore the net efficiency is high.

(v) The electron temperatures in the two cases are different. For $\dot{m} \sim \dot{m}_{\text {crit }}$, accreting black holes reach $T_{e} \sim 10^{9}-10^{9.5} \mathrm{~K}$ at small radii, whereas accreting neutron star flows saturate 
at $T_{e} \sim 10^{8.5}-10^{9} \mathrm{~K}$. For $\dot{m} \ll \dot{m}_{\text {crit }}$, the temperatures in the black hole systems are even higher, $T_{e} \gtrsim 10^{10} \mathrm{~K}$, but the neutron star systems hardly ever exceed $T_{e} \sim 10^{9} \mathrm{~K}$. As before, the difference between the two kinds of systems is because of the additional cooling in the neutron star case due to Comptonization of the stellar photons. A robust prediction therefore is that black hole systems will have harder spectra, as appears to be the case in some of the observations.

(vi) Given the high electron temperatures in our models, we may expect pair effects to become important. It turns out that this is not an issue for any of the models described in this paper. Our estimates of the equilibrium positron fraction, calculated using the relations given in White \& Lightman (1989, see also Kusunose \& Takahara 1989), are never larger than $\sim 10^{-3}$. However, at higher accretion rates than those we have considered, i.e. for $\dot{m}>\dot{m}_{\text {crit }}$, the optical depth will increase rapidly and it is quite possible that various kinds of pair instabilities may occur. Based on the fact that our black hole models are invariably hotter than the neutron star models, we think that perhaps pair effects will be most significant for accreting black holes at high $\dot{m}$. We further feel that accreting neutron stars may never reach the required temperatures for copious pair production at any value of $\dot{m}$. Observationally, we note that pair annihilation lines have been seen only from black hole candidates and not from any confirmed neutron star system.

In discussions of AGN, a spherical accretion model has been discussed sometimes in the literature (e.g. McCray 1979, Ipser \& Price 1983, Melia 1994). In this model, the angular momentum of the accreting gas is completely neglected, a rather severe approximation. Although our advection-dominated solution is quasi-spherical (see Paper 2) it does include differential rotation, viscosity, angular momentum transport, etc., all in a self-consistent fashion. We may therefore describe our models as more realistic and self-consistent versions of the idealized spherical accretion models discussed in the past.

In future papers we intend to discuss the radiation emitted by our advection-dominated flows and to compare model spectra with observations of low- $\dot{m}$ black hole and neutron star systems. The information in Fig. 7 indicates what we may qualitatively expect for the spectrum. Since the bulk of the emission in an accretion flow comes from small radii, the spectrum will be dominated by synchrotron emission and Comptonization of the synchrotron photons. The primary synchrotron emission in the models will give a peak in the spectrum at the self-absorption frequency $\nu_{c}$ (equation 3.15). The position of the peak depends on the particular values of the various parameters, $M, \dot{M}, \alpha, \beta$, but roughly we expect the synchrotron peak to occur at $\nu \sim 10^{15} \mathrm{~Hz}$ for a stellar-mass object and at $\nu \sim 10^{11} \mathrm{~Hz}$ for a $10^{8} M_{\odot}$ black hole. The Comptonization of the synchrotron emission will produce one or more bumps in the spectrum at higher frequencies, or a near power-law spectrum if the Compton $y$-parameter is large enough. Rather interestingly, we see from Fig. 7 that Comptonization is quite important for a range of accretion rates from $\dot{m} \sim \dot{m}_{\text {crit }}$ down to $\dot{m} \sim 0.01 \dot{m}_{\text {crit }}$. Finally, the spectrum will have a peak at a frequency $\nu \sim 10^{20} \mathrm{~Hz}$ (i.e. at a photon energy of order a few hundred keV), due to bremsstrahlung emission by the hot electrons.

Acknowledgements: We thank D. Barret, J. Grindlay, J.-P. Lasota, E. Liang, R. Mahadevan and J. Ostriker for useful discussions, and M. Abramowicz, R. Blandford, D. Eardley, A. Lightman, S. Phinney, M. Rees and S. Shapiro for comments. We are grateful to our second referee, Dr. R. E. Taam, for prompt arbitration after the paper was rejected by the first referee, and for making a number of very constructive suggestions. This work was supported in part by grant AST9148279 from the National Science Foundation. 


\section{Appendix A: Non-Thermal Coupling of Ions and Electrons}

Throughout this paper we have assumed that the only coupling between ions and electrons is via Coulomb collisions. This assumption leads to a two-temperature plasma in the inner regions of our solutions, and it is the two-temperature feature which allows our flows to be advection-dominated. However, it has been argued (cf. Phinney 1981) that, in addition to Coulomb collisions, many other non-thermal processes may couple ions and electrons efficiently and these processes could potentially eliminate the two-temperature structure. This is an important issue for our work since most of the properties of our solutions are a consequence of the two-temperature nature of the plasma.

In the astrophysical literature, the only work we are aware of discussing a specific nonthermal mechanism to heat electrons is that of Begelman \& Chiueh (1989, hereafter BC). $\mathrm{BC}$ start with the interesting fact that a two-temperature plasma has the following unusual ordering of lengthscales,

$$
\lambda_{c e}<\lambda_{D e} \ll \lambda_{D i}<\lambda_{c i},
$$

where $\lambda_{D e}, \lambda_{D i}$ are the electron and ion Debye lengths, and $\lambda_{c e}, \lambda_{c i}$ are the respective gyroradii. $\mathrm{BC}$ then show that, under these conditions, plasma waves traveling through a suitably turbulent zone can pump energy directly into the electrons through collective motions. The energy transfer via this mechanism may, in principle, occur rapidly enough to eliminate the two-temperature structure of the plasma.

We calculate here the rate of heating of electrons via the $\mathrm{BC}$ mechanism for the specific flows considered in this paper, and compare this rate to the rate of energy tranfer from ions to electrons via Coulomb collisions. For simplicity, we set $\beta=0.5$ (equipartition fields), $f=1$ (fully advection-dominated flow), and correspondingly choose $c_{1}=0.489, c_{2}=0.429, c_{3}=$ $0.326, \epsilon=\epsilon^{\prime}=0.565$. Also, we set $T_{e} \ll T_{i}$ in equation (2.16), so that $T_{i}$ is equal to the right-hand side of this equation.

From the expression for $q^{+}$in equation (2.15), we find that the rate at which an ion is heated by viscous dissipation is given by

$$
\left(\frac{d E_{i}}{d t}\right)_{v i s}=8.95 \alpha m^{-1} r^{-5 / 2} \mathrm{erg} \mathrm{s}^{-1} .
$$

Assuming that the ions are non-relativistic and the electrons are relativistic, equation (3.3) for the rate of heating of electrons by Coulomb collisions simplifies to

$$
\left(\frac{d E_{e}}{d t}\right)_{\text {Coul }}=5.9 \alpha m^{-1}\left(\frac{\dot{m}}{\alpha^{2}}\right) r^{-5 / 2} T_{10}^{-1} \operatorname{erg~s}^{-1},
$$

where we have written $T_{e}=10^{10} T_{10} \mathrm{~K}$. Recall that, because of the efficient cooling of the electrons, any energy transferred to the electrons is immediately radiated. Therefore, for an advection-dominated solution, we require $\left(d E_{e} / d t\right)_{C o u l}<\left(d E_{i} / d t\right)_{v i s}$. From equations (A2) and (A3), this gives the condition

$$
\left(\frac{\dot{m}}{\alpha^{2}}\right)<\left(\frac{\dot{m}}{\alpha^{2}}\right)_{\text {crit }} \sim 1.5 T_{10} .
$$


This scaling is consistent with the detailed numerical results for $\dot{m}_{c r i t}$ shown in Fig. 3 for the inner regions of accreting black holes.

According to BC, the rate of heating of electrons when their instability is fully developed is given by

$$
\left(\frac{d E_{e}}{d t}\right)_{B C} \sim f_{B C} \frac{m_{i} v_{t i}^{3}}{\lambda_{D i}}\left(\frac{v_{A}}{c}\right)^{2}\left(\frac{\lambda_{c i}}{L}\right)^{9 / 2},
$$

where $f_{B C}$ is the filling factor of the plasma where the kind of strong turbulence needed for the instability is present, $m_{i}$ is the mass of the ions (which we take to be protons), $v_{t i}$ is the ion thermal velocity, $v_{A}$ is the Alfven velocity, and $L$ is the local density gradient scale. For the self-similar scalings given in equation (2.15), we have

$$
\begin{gathered}
v_{t i}=8.56 \times 10^{9} r^{-1 / 2} \mathrm{~cm} \mathrm{~s}^{-1} \\
\lambda_{D i}=8.84 \times 10^{-4} \alpha^{-1 / 2} m^{1 / 2}\left(\frac{\dot{m}}{\alpha^{2}}\right)^{-1 / 2} r^{1 / 4} \mathrm{~cm} \\
v_{A}=1.21 \times 10^{10} r^{-1 / 2} \mathrm{~cm} \mathrm{~s}^{-1} \\
\lambda_{c i}=3.13 \times 10^{-3} \alpha^{-1 / 2} \mathrm{~m}^{1 / 2}\left(\frac{\dot{m}}{\alpha^{2}}\right)^{-1 / 2} r^{3 / 4} \mathrm{~cm} .
\end{gathered}
$$

The values of $f_{B C}$ and $L$ depend on the particular turbulence scenario we consider. We discuss two limits below.

\section{Kolmogorov-Like Turbulent Cascade}

Let us suppose we have a space-filling turbulent cascade, and let us assume that it satisfies the Kolmogorov scaling, with an outer scale equal to the disk thickness $H$. Then, following equation (5.9) of $\mathrm{BC}$, the gradient scale $L$ satisfies

$$
\left(\frac{L}{\lambda_{c i}}\right) \sim 100 \alpha^{1 / 6} m^{-1 / 6}\left(\frac{\dot{m}}{\alpha^{2}}\right)^{-1 / 6} r^{-1 / 12} .
$$

The filling factor $f_{B C}$ is potentially equal to unity in the case of a turbulent cascade, but we retain it as a factor since it is possible that only a fraction of the volume actually satisfies all the conditions required for the BC instability. (For instance, subsonic turbulence is usually incompressible and it is not clear that it will have sufficient density contrasts for the BC mechanism to operate.) Then, the rate of heating of electrons by the BC mechanism is given by

$$
\left(\frac{d E_{e}}{d t}\right)_{B C} \sim 0.21 \alpha^{-1 / 4} m^{-5 / 4}\left(\frac{\dot{m}}{\alpha^{2}}\right)^{-1 / 4} r^{-25 / 8} \mathrm{erg} \mathrm{s}^{-1} .
$$

Now, BC have shown that their instability operates only if the scale $L$ satisfies a particular inequality (cf. equation 5.6 in their paper). For our choice of $\beta$, the relevant condition is

$$
1<\frac{L}{\lambda_{c i}}<2.2 r^{1 / 2}
$$


Comparing this condition with equation (A10), we see that the BC instability operates only for radii $r$ greater than a certain limit:

$$
r>r_{\text {crit }} \sim 690 \alpha^{2 / 7} m^{-2 / 7}\left(\frac{\dot{m}}{\alpha^{2}}\right)^{-2 / 7} .
$$

Let us substitute $r=r_{\text {crit }}$ into equation (A11). We then find that the electron heating rate at the optimum radius satisfies

$$
\frac{\left(d E_{e} / d t\right)_{B C, \max }}{\left(d E_{e} / d t\right)_{\text {Coul }}} \sim 6.0 \times 10^{-4} f_{B C} \alpha^{-10 / 7} m^{-1 / 14}\left(\frac{\dot{m}}{\alpha^{2}}\right)^{-15 / 14} .
$$

The right-hand side of (A14) is less than unity under most conditions of interest for our solutions. This shows that Coulomb heating usually dominates. Only for very small values of $\alpha$ or very small mass accretion rates does the $\mathrm{BC}$ heating become competitive with Coulomb heating. Even when the heating ratio (A14) becomes comparable to or larger than unity, the ratio $\left(d E_{i} / d t\right)_{v i s} /\left(d E_{e} / d t\right)_{B C}$ is usually much larger than unity except when $\alpha<4 \times 10^{-3}$. In other words, unless $\alpha$ becomes very small, the viscous heating of ions dominates over the Begelman-Chiueh heating and a two-temperature plasma is very likely. We note that this conclusion is only very weakly dependent on $m$ and $\dot{m}$.

\section{Collisionless Shocks}

The other possibility is that the turbulent gas may have some fraction of the dissipation occurring through collisionless shocks. In the case of shocks, we expect the gradient scale $L$ to be of order the ion gryroradius $\lambda_{c i}$, so that the condition (A10) is easily satisfied. To calculate the filling factor of the shock fronts, we note that the volume rate of dissipation of energy within a shock is approximately

$$
q_{s h} \sim \frac{\rho v_{A}^{2}}{\lambda_{c i} / v_{A}} \sim 2.6 \times 10^{28} \alpha^{-3 / 2} m^{-3 / 2}\left(\frac{\dot{m}}{\alpha^{2}}\right)^{3 / 2} r^{-15 / 4} \mathrm{erg} \mathrm{cm}^{-3} \mathrm{~s}^{-1} .
$$

Since the total dissipation rate per unit volume is $q^{+}$, we can calculate the maximum filling factor $f_{B C, \max }$ of the shocks by equating $f_{B C, \max } q_{s h}$ to $q^{+}$:

$$
f_{B C, \max } \sim 2.3 \times 10^{-8} \alpha^{5 / 2} m^{-1 / 2}\left(\frac{\dot{m}}{\alpha^{2}}\right)^{1 / 2} r^{-1 / 4} .
$$

Let us write $f_{B C}=\delta f_{B C, \max }$, so that the actual volume fraction which participates in the $\mathrm{BC}$ mechanism is only a fraction $\delta$ of the maximum allowed. Then we obtain

$$
\frac{\left(d E_{e} / d t\right)_{B C}}{\left(d E_{e} / d t\right)_{\text {Coul }}} \sim 0.74 \delta \alpha^{2} m r^{1 / 2} T_{10}
$$

We see that the BC mechanism may be quite important in the case of shocks, whereas it is irrelevant in the case of the Kolmogorov cascade considered earlier. Note the curious fact that $\dot{m}$ does not enter in equation (A17). For $\alpha \sim 0.1$ and $r \lesssim 10^{2}$ (cf. the two-temperature zone in Fig. 3), we see that the BC mechanism via shocks becomes more important than Coulomb transfer if $\delta m>14$. We have no idea what a reasonable estimate of $\delta$ may be, but we see that even if $\delta \sim 1$, a two-temperature gas is allowed for flows around stellar-mass black holes. Thus, the models developed in this paper are likely to be valid for Galactic X-ray binaries. However, it appears that these solutions may run into difficulties for AGN models, unless $\delta$ is extremely small. A small $\delta$ requires either negligible dissipation through shocks or an inefficient $\mathrm{BC}$ mechanism in the vicinity of shocks. 


\section{Appendix B: Radiative Viscosity}

In our models we have assumed an $\alpha$ form of viscosity which implicity assumes that the viscous stress has a radius-independent self-similar form. Such a viscosity could arise from magnetic stresses or turbulent stresses (Shakura \& Sunyaev 1973). There has been some discussion in the literature of radiative viscosity due to the scattering of photons off electrons, both in the context of black hole flows (Loeb \& Laor 1992) and neutron star flows (Miller \& Lamb 1993). One feature of radiative viscosity is that the effective $\alpha$ due to it depends on the radius. Therefore, if this viscosity is important, we will need to modify the scalings we have given in equation (2.15). We discuss here the magnitude of $\alpha_{\text {rad }}$ due to radiation drag for the black hole and neutron star problems.

Because we have flows which are optically thin to infinity, especially at low $\dot{m}$, some of the standard formulae in the literature for the radiative viscosity are not appropriate. If the luminosity of the accreting system is $L$, then we expect the radiation density $u_{p h}$ at radius $R$ to be of order

$$
u_{p h} \sim \frac{L}{4 \pi R^{2} c}=\frac{\eta \dot{M} c}{4 \pi R^{2}},
$$

where $\eta$ is the radiative efficiency of the accretion flow. The probability per unit time that a given photon scatters is $n_{e} \sigma_{T} c$ and on average the post-scattered photon will have a tangential velocity $\sim \Omega R$. Thus, the rate at which angular momentum is removed from the accreting gas per unit volume per unit time is

$$
\dot{J} \sim R \frac{u_{p h} \Omega R}{c^{2}} n_{e} \sigma_{T} c \sim \frac{L \Omega n_{e} \sigma_{T}}{4 \pi c^{2}} .
$$

The effective $\alpha_{\text {rad }}$ due to this radiative viscosity is obtained by dividing $\dot{J}$ by the gas pressure $p$. Thus

$$
\alpha_{\text {rad }} \sim \frac{\eta \dot{M} \Omega n_{e} \sigma_{T}}{4 \pi p} .
$$

As in Appendix A, let us set $\beta=0.5, f \rightarrow 1$ and use the corresponding values of $c_{1}-c_{3}$. As described in the main paper, the efficiency of our advection-dominated flows is approximately given by $\eta \sim 0.1\left(\dot{m} / \alpha^{2}\right)$ in the case of a black hole and $\eta \sim 0.2$ in the case of a neutron star. We thus find

$$
\alpha_{\text {rad }} \sim 0.8 \alpha^{2}\left(\frac{\dot{m}}{\alpha^{2}}\right)^{2} r^{-1 / 2}
$$

in the case of an accreting black hole, and

$$
\alpha_{\text {rad }} \sim 1.6 \alpha^{2}\left(\frac{\dot{m}}{\alpha^{2}}\right) r^{-1 / 2}
$$

in the case of an accreting neutron star. We expect $\alpha^{2} \lesssim 0.1$. Also, for advection-dominated flows, $\dot{m} / \alpha^{2}<1$ for a black hole and $\dot{m} / \alpha^{2}<0.1$ for a neutron star. Therefore, radiative viscosity is not likely to be important for our optically-thin advection-dominated flows except perhaps very close to the center. 


\section{References}

Abramowicz, M., Czerny, B., Lasota, J. P., \& Szuszkiewicz, E. 1988, ApJ, 332, 646

Abramowicz, M., Chen, X., Kato, S., Lasota, J. P., \& Regev, O. 1995, ApJ, Jan 1

Adam, S., Stoerzer, H., Wehrse, R., \& Shaviv, G. 1986, A\&A, 193, L1

Balbus, S. A. \& Hawley, J. F. 1991, ApJ, 376, 214

Begelman, M. C. 1978, MNRAS, 184, 53

Begelman, M. C. \& Chiueh, T. 1988, ApJ, 332, 872

Begelman, M. C. \& Meier, D. L. 1982, ApJ, 253, 873

Chen, X. 1995, preprint

Chen, X. \& Taam, R. E. 1993, ApJ, 412, 254

Clayton, D. D. 1983, Principles of Stellar Evolution and Nucleosynthesis, (Chicago: The University of Chicago Press)

Coroniti, F. V. 1981, ApJ, 244, 587

Dermer, C. D., Liang, E. P., \& Canfield, E. 1991, ApJ, 369, 410

Eardley, D. M., Lightman, A. P., Payne, D. G., \& Shapiro, S. L. 1978, ApJ, 224, 53

Eggum, G. E., Coroniti, F. V., \& Katz, J. I. 1988, ApJ, 330, 142

Frank, J., King, A., \& Raine, D. 1992, Accretion Power in Astrophysics (Cambridge, UK: Cambridge University Press)

Grindlay, J. E. 1978, ApJ, 221, 234

Honma, F., Matsumoto, R., \& Kato, S. 1991, PASJ, 43, 147

Hubeny, I. 1990, ApJ, 351, 632

Ipser, J. R. \& Price, R. H. 1983, ApJ, 267, 371

Kusunose, M. \& Takahara, F. 1989, PASJ, 41, 263

Liang, E. P. 1988, ApJ, 334, 339

Liang, E. P. 1993, Proc. Compton Gamma-Ray Observatory Workshop

Liang, E. P. T. \& Thompson, K. A. 1980, ApJ, 240, 271

Lightman, A. P. \& Eardley, D. M. 1974, ApJ, 187, L1

Loeb, A. \& Laor, A. 1992, ApJ, 384, 115

Luo, C. \& Liang, E. P. 1994, MNRAS, 266, 386

Lynden-Bell, D. \& Pringle, J. E. 1974, MNRAS, 168, 603

Mahadevan, R., Narayan, R., \& Yi, I. 1994, in preparation

McCray, R. 1979, in Active Galactic Nuclei, ed. C. Hazard \& S. Mitton (Cambridge, UK: Cambridge University Press)

Melia, F. 1994, ApJ, 426, 577

Melia, F. \& Misra, R. 1993, ApJ, 411, 797

Meyer, F. \& Meyer-Hofmeister, E. 1994, A\&A, 288, 175

Miller, M. C. \& Lamb, F. K. 1993, ApJ, 413, L43

Mineshige, S. \& Wheeler, J. C. 1989, ApJ, 343, 241

Muchotrzeb, B. \& Paczyński, B. 1982, Acta Astron., 32, 1

Narayan, R. \& Popham, R. 1993, Nature, 362, 820

Narayan, R. \& Yi, I., 1994, ApJ, 428, L13 (Paper 1)

Narayan, R. \& Yi, I., 1995, ApJ, in press (Paper 2)

Novikov, I. D. \& Thorne, K. S. 1973, in Blackholes ed. C. DeWitt \& B. DeWitt (New York: Gordon and Breach)

Ostriker, J. P., McCray, R., Weaver, R., \& Yahil, A. 1976, ApJ, 208, L61

Pacholczyk, A. G. 1970, Radio Astrophysics (San Francisco: Freeman)

Paczyński, B. \& Bisnovatyi-Kogan, G. 1981, Acta Astr., 31, 283 
Paczyński, B. \& Wiita, P. J. 1980, A\&A, 88, 23

Phinney, E. S. 1981, in Plasma Astrophysics. ESA SP-161, Paris: European Space Agency Piran, T. 1978, ApJ, 221, 652

Popham, R. \& Narayan, R. 1995, ApJ, in press

Popham, R., Narayan, R., Hartmann, L., \& Kenyon, S. 1993, ApJ, 415, L127

Pringle, J. E., Rees, M. J., \& Pacholczyk, A. G. 1973, A\&A, 29, 179

Rees, M. J., Begelman, M. C., Blandford, R. D., \& Phinney, E. S. 1982, Nature, 295, 17

Shakura, N. I. \& Sunyaev, R. A. 1973, A\&A, 24, 337

Shapiro, S. L., Lightman, A. P., \& Eardley, D. M. 1976, ApJ, 204, 187 (SLE)

Shaviv, G. \& Wehrse, R. 1986, A\&A, 159, L5

Shaviv, G. \& Wehrse, R. 1989, in Theory of Accretion Disks, eds F. Meyer et al., p419 (Dordrecht: Kluwer)

Smak, J. 1984, PASP, 96, 5

Spruit, H. C., Matsuda, T., Inoue, M., \& Sawada, K. 1987, MNRAS, 229, 517

Stella, L. \& Rosner, R. 1984, ApJ, 277, 312

Stepney, S. \& Guilbert, P. W. 1983, MNRAS, 204, 1269

Sunyaev, R. A., et al. 1991a, in Proc. 28th Yamada Conf. on Front. X-ray Astr. (Nagoya, Japan)

Sunyaev, R. A., et al. 1991b, A\&A, 247, L29

Sunyaev, R. A. \& Titarchuk, L. G. 1980, A\&A, 86, 127

Svensson, R. 1982, ApJ, 258, 335

Svensson, R. 1984, MNRAS, 209, 175

Wallinder, F. H. 1991, A\&A, 249, 107

Wandel, A. \& Liang, E. P. 1991, ApJ, 380, 84

White, T. R. \& Lightman, A. P. 1989, ApJ, 340, 1024

Zdziarski, A. A. 1985, ApJ, 289, 514 


\section{Figure Captions}

Figure 1. The solid line shows at each scaled radius $r$ the scaled mass accretion rate $\dot{m}$ at which $f=0.5$, i.e. the $\dot{m}$ at which exactly half the dissipated energy is radiated and half is advected. The case shown corresponds to a $10 M_{\odot}$ black hole with $\alpha=0.3, \beta=0.5$, and the curve has been obtained by solving the full equations of $\S \S 2,3$. The dotted line shows the approximate relation (4.1) derived under the simplifying assumptions that the ion and electron temperatures are equal and that bremsstrahlung cooling dominates.

Figure 2. Shows the three solution branches for an accreting $10 M_{\odot}$ black hole at a radius of $r=10^{3}$. The uppermost branch is the new advection-dominated branch discussed in this paper. Note that $f \rightarrow 1$ for this branch, corresponding to most of the dissipated energy being advected with the flow. The lowermost branch corresponds to the standard coolingdominated $(f \rightarrow 0)$ thin accretion disk solution. These two stable branches are connected by an unstable middle branch, indicated by a dotted line, which corresponds to the hot solution discovered originally by SLE. See Fig. 8 for more details.

Figure 3. Solid lines in the upper three panels show the critical accretion rate, $\dot{m}_{\text {crit }}$, above which the advection-dominated branch ceases to exist. Three values of $\alpha$ are considered: $0.03,0.1,0.3$. The advection parameter $f$ is $\sim 0.3$ on these lines. The dotted lines correspond to $f=0.9$ where $90 \%$ of the dissipated energy is advected. The bottom three panels show the variations of the ion temperature $T_{i}$ and electron temperature $T_{e}$ along the lines in the upper panels. Note that the accreting plasma is single temperature and virial at large radii $r \gtrsim 10^{3}$. For $r \lesssim 10^{3}, T_{i}$ remains nearly virial but $T_{e}$ saturates because of poor coupling between the ions and the electrons plus the availability of a variety of efficient cooling mechanisms for the electrons.

Figure 4. Comparison of the critical lines $\dot{m}_{c r i t}(r)$ (upper panel) and the ion and electron temperatures (lower panel) for models of two accreting black holes of masses $10 M_{\odot}$ (solid lines) and $10^{8} M_{\odot}$ (dashed lines). Note that the two models give virtually identical results, showing that advection-dominated flows around black holes are essentially mass-independent.

Figure 5. Similar to Fig. 3 but for an accreting neutron star with scaled mass $m=1.4$ and radius $r_{*}=2.5$ (corresponding to a stellar radius of $10.5 \mathrm{~km}$ ). The main new feature in these models is that the advected energy is assumed to be thermalized and radiated from the stellar surface. This radiation provides an important source of Compton cooling for the accreting gas. Note that the critical lines $\dot{m}_{c r i t}$ for these models are significantly lower than in Fig. 3, and that the electron temperature is also lower. In panels $a$ and $b$, the radiation from the neutron star is taken to be a fully thermalized blackbody, while in panel $c$ it is assumed to be at a temperature of $10^{9} \mathrm{~K}$.

Figure 6. Indicates the relative importance of the various cooling mechanisms for two black hole models $\left(10 M_{\odot}\right.$ in panel a and $10^{8} M_{\odot}$ in panel b) and a neutron star model (panel c). Bremsstrahlung cooling $\left(q_{b r}^{-}+q_{b r, C}^{-}\right)$is shown by dotted lines, synchrotron cooling $\left(q_{\text {synch }}^{-}+\right.$ $\left.q_{\text {synch }, C}^{-}\right)$is shown by short-dashed lines, and Compton cooling by soft stellar photons $\left(q_{*, C}^{-}\right)$ is indicated by long-dashed lines. The various cooling terms were computed along the critical 
lines $\dot{m}_{c r i t}(r)$ in Figs. $3-5$. In the black hole models bremsstrahlung dominates at large radii and synchrotron at small radii. In the neutron star model, Comptonization of stellar photons dominates out to $r \sim 10^{5}$.

Figure 7 . Indicates the zones in the $\dot{m} r$ plane of an accreting $10 M_{\odot}$ black hole where various cooling terms dominate. Zone 1 corresponds to the region where bremsstrahlung cooling (whose volume rate is given by $q_{b r}^{-}$) dominates, zone 2 to Comptonized bremsstrahlung $\left(q_{b r, C}^{-}\right)$, zone 3 to synchrotron emission $\left(q_{\text {synch }}^{-}\right)$, and zone 4 to Comptonized synchrotron $\left(q_{\text {synch,C }}^{-}\right)$. Note that bremsstrahlung-related emission dominates at large $r$ and synchrotronrelated emission at small $r$, while Comptonization of these is important at high $\dot{m}$.

Figure 8. (a) Shows the nature of the three solutions, marked A, B, C, for a typical case consisting of a $10 M_{\odot}$ black hole accreting with $\dot{m}=10^{-3}$. The situation shown corresponds to a radius $r=10^{3}$. Solution A corresponds to a standard thin accretion disk solution. Because $T_{i}$ increases with increasing $q^{-} /(1-f) q^{+}$, this is a stable solution as discussed in the text. Solution $\mathrm{C}$ is our new advection-dominated solution and is again stable. Solution $\mathrm{B}$ is, however, an unstable solution as a result of the wrong sign of the slope. Because the two stable solutions A, C bracket the unstable solution B, the gas can find a thermally stable configuration starting from any initial configuration. (b) Similar to panel a, but with advection ignored. Note that solution $\mathrm{C}$ disappears. If this gas is set up with an initial $T_{i} \gtrsim 10^{7.5} \mathrm{~K}$, then it will heat up without limit and will be unable to find a thermally stable equilibrium. This paradoxical situation is the result of ignoring advection. (c) Similar to Fig. 2 , but showing $T_{i}$ along the vertical axis instead of $f$. The branches are labeled $\mathrm{A}, \mathrm{B}, \mathrm{C}$, as in panel a. Note that $\mathrm{B}$ and $\mathrm{C}$ both correspond to hot solutions. $\mathrm{B}$ is the usual hot solution discussed in the literature, discovered originally by SLE, while $\mathrm{C}$ is the new advectiondominated hot solution of this paper. (d) Another representation, where the vertical axis shows $\dot{m}$ and the horizontal axis shows the surface density $\Sigma$. Note the characteristic Scurve. Note also that, for all $\dot{m}$, there is at least one stable solution available. Therefore, this gas will not undergo thermal limit cycle behavior. The shape of the S-curve in this diagram is different from that in other situations where a limit cycle is argued to occur. If radiation pressure effects are included (which are ignored in our calculations), then the A branch will develop another S curve near the top right corner of the panel (see Abramowicz et al. 1988) because of the Lightman-Eardley (1974) instability.

Figure 9. (a) The dashed line shows the boundary above which the Lightman-Eardley (1974) instability of a radiation-dominated thin accretion disk operates. The case shown corresponds to an accreting $10 M_{\odot}$ black hole. The solid line shows $\dot{m}_{c r i t}(r)$. In the small triangular region between the two lines, the thin disk solution is unstable but the advectiondominated solution is stable. Advection-dominated accretion must occur in this region. (b) Equivalent results for a $10^{8} M_{\odot}$ black hole.

Figure 10. (a) The dotted line indicates the track of a $10 M_{\odot}$ black hole accreting at a rate $\dot{m}=10^{-2}$. The solid circle indicates a critical radius $r_{\text {crit }}=10^{5.5}$ such that for $r<r_{\text {crit }}$ the track is located in a region where advection-dominated accretion is allowed $\left(\dot{m}<\dot{m}_{\text {crit }}\right)$ whereas for $r>r_{\text {crit }}$ only thin disk accretion is possible $\left(\dot{m}>\dot{m}_{\text {crit }}\right)$. If the accretion flow begins with an outer radius $r_{\text {out }}<r_{\text {crit }}$ and if it is initially in a nearly virial state, then it will accrete in an advection-dominated mode all the way down to the horizon. (b) 
Shows a case where $r_{\text {out }}>r_{\text {crit }}$. We propose that the accreting material forms a disk plus a corona with the indicated mass accretion rates, $\dot{m}_{d i s k}$ and $\dot{m}_{c o r}$, in the region $r>r_{c r i t}$. For $r<r_{c r i t}$ we propose that the gas switches completely to the advection-dominated mode.

Figure 11. The solid line in the panel on the left shows the variation of the radiated luminosity of an accreting $10 M_{\odot}$ black hole as a function of the mass accretion rate $\dot{M}$, for $\alpha=0.3, \beta=0.5$. The dashed line shows the dependence expected if the radiative efficiency is $\eta=0.1$, i.e. if $L=0.1 \dot{M} c^{2}$. The actual efficiency is lower, especially at low accretion rates, because the flow is advection-dominated. The panel on the right corresponds to an accreting neutron star. In this case, the net radiative efficiency is $\eta \sim 0.2$ regardless of $\dot{M}$. However, most of the energy is advected with the accretion flow and radiated at the stellar surface. The luminosity radiated by the accretion flow itself is shown by the dotted line, which is seen to have a low efficiency. 
This figure "fig1-1.png" is available in "png" format from: http://arXiv.org/ps/astro-ph/9411059v2 
This figure "fig2-1.png" is available in "png" format from: http://arXiv.org/ps/astro-ph/9411059v2 
This figure "fig3-1.png" is available in "png" format from: http://arXiv.org/ps/astro-ph/9411059v2 
This figure "fig4-1.png" is available in "png" format from: http://arXiv.org/ps/astro-ph/9411059v2 
This figure "fig1-2.png" is available in "png" format from: http://arXiv.org/ps/astro-ph/9411059v2 
This figure "fig2-2.png" is available in "png" format from: http://arXiv.org/ps/astro-ph/9411059v2 
This figure "fig3-2.png" is available in "png" format from: http://arXiv.org/ps/astro-ph/9411059v2 
This figure "fig4-2.png" is available in "png" format from: http://arXiv.org/ps/astro-ph/9411059v2 
This figure "fig1-3.png" is available in "png" format from: http://arXiv.org/ps/astro-ph/9411059v2 
This figure "fig2-3.png" is available in "png" format from: http://arXiv.org/ps/astro-ph/9411059v2 